\title{
RTL Functional Test Generation using Factored Concolic Execution
}

\author{
Sonal Pinto \\ Thesis submitted to the Faculty of the \\ Virginia Polytechnic Institute and State University \\ in partial fulfillment of the requirements for the degree of \\ Master of Science \\ in \\ Computer Engineering \\ Michael S. Hsiao, Chair \\ Haibo Zeng \\ Patrick R. Schaumont \\ June 1, 2017 \\ Blacksburg, Virginia
}

Keywords: Functional Test Generation, Control-Flow Response, Concolic Execution, Branch Coverage, System State

Copyright 2017, Sonal Pinto 


\title{
RTL Functional Test Generation using Factored Concolic Execution
}

\author{
Sonal Pinto
}

(ABSTRACT)

This thesis presents a novel concolic testing methodology and CORT, a test generation framework that uses it for high-level functional test generation. The test generation effort is visualized as the systematic unraveling of the control-flow response of the design over multiple (factored) explorations. We begin by transforming the Register Transfer Level (RTL) source for the design into a high-performance $\mathrm{C}++$ compiled functional simulator which is instrumented for branch coverage. An exploration begins by simulating the design with concrete stimuli. Then, we perform an interleaved cycle-by-cycle symbolic evaluation over the concrete execution trace extracted from the Control Flow Graph (CFG) of the design. The purpose of this task is to dynamically discover means to divert the control flow of the system, by mutating primary-input stimulated control statements in this trace. We record the control-flow response as a Test Decision Tree (TDT), a new representation for the test generation effort. Successive explorations begin at system states heuristically selected from a global TDT, onto which each new decision tree resultant from an exploration is stitched. CORT succeeds at constructing functional tests for ITC99 and IWLS-2005 benchmarks that achieve high branch coverage using the fewest number of input vectors, faster than existing methods. Furthermore, we achieve orders of magnitude speedup compared to previous hybrid concrete and symbolic simulation based techniques.

This work was supported in part by the DARPA CRAFT Verification Task (DARPA-BAA15-55) and the National Science Foundation Project 1422054. 


\title{
RTL Functional Test Generation using Factored Concolic Execution
}

\author{
Sonal Pinto
}

(GENERAL AUDIENCE ABSTRACT)

In recent years, the cost of verifying digital designs has outpaced the cost of development, in terms of both resources and time. The scale and complexity of modern designs have made it increasingly impractical to manually verify the design. In the process of circuit design, designers use Hardware Descriptive Languages (HDL) to abstract the design in a manner similar to software programming languages. This thesis presents a novel methodology for the automation of testing functional level hardware description with the aim of maximizing branch coverage. Branches indicate decision points in the design, and tests with high branch coverage are able to thoroughly exercise the design in a manner that randomly generated tests cannot. In our work, the design is simulated concretely with a random test (a sequence

of input or stimulus). During simulation, we analyze the flow of behavioral statements and decisions executed to construct a formulaic interpretation of the design execution in terms of syntactical elements, to uncover differentiating input that could have diverted the flow of execution to unstimulated parts of the design. This process is formally known as Concolic Execution. The techniques described in this thesis tightly interleaves concrete and symbolic simulation (concolic execution) of hardware designs to generate tests with high branch coverage, orders of magnitude faster than previous similar work. 
$\sim$ To my family $\sim$ 


\section{Acknowledgments}

This work would be incomplete without acknowledging the guidance and support of those who made it possible. To begin with, I owe my greatest thanks to my research mentor, Dr. Michael S. Hsiao for granting me the opportunity to engage in invigorating research and to participate as his research assistant. His excellent course on "Electronic Design Automation" in Spring 2016 piqued my curiosity and inspired me to pursue my Master's thesis under his tutelage. Throughout the span of the work that went into this thesis, he tirelessly guided and polished my work with his extensive background and experience in the field. I am honored to have received a chance to work with him.

I would like to thank Dr. Haibo Zeng and Dr. Patrick Schaumont for graciously agreeing to serve on my thesis committee. I also thank the administrative staff of the Graduate School and the Bradley Department of Electrical and Computer Engineering for their timely help and continued cooperation with paperwork and other administrative matters.

I am thankful to my friends in the PROACTIVE Research Group for enriching my graduate school experience: Tonmoy Roy, Kunal Bansal, Yue Zhan, Aravind V., Tania Khanna and ChungHa Sung. I am especially grateful to Tonmoy for his patience in engaging me in my animated narrations of knowledge that I gleaned from the research literature. He introduced me to the complex domain of symbolic execution in hardware and entertained every query I had on the topic. 
I thank Shobek Sam, Shobal Sam, Shriya Shah, Nahush Gondhalekar, Harsh Patel and Akshatha Kini for indeed making my graduate school experience unforgettable. I am thankful for the love and support of my family, without whom I would not even have had the opportunity to experience a fulfilling graduate school life at Virginia Tech. Lastly, I am thankful to my loving fiancee, Rohita Darga, for believing in me, and being my ultimate pillar of support and ensuring that I didn't starve.

Sonal Pinto

Blacksburg

June 1,2017 


\section{Contents}

List of Figures $\quad$ ix

List of Tables $\quad$ x

List of Algorithms $\quad$ xi

1 Introduction $\quad 1$

1.1 Contributions of this Thesis . . . . . . . . . . . . . . 2

1.2 Thesis Organization . . . . . . . . . . . . . . . . 4

2 Background $\quad 5$

2.1 Automated Functional Test Generation . . . . . . . . . . . . . . . 5

2.2 Concolic Execution . . . . . . . . . . . . . . . . . 8

2.3 Scaling through Factored Exploration . . . . . . . . . . . . . . . 10

2.4 Verilator and RTL Instrumentation . . . . . . . . . . . . . . 15

2.5 Related Work . . . . . . . . . . . . . . . . . . . 17

3 Cycle-by-Cycle Concolic Execution of RTL 20

3.1 Terminology . . . . . . . . . . . . . . . . . . . . 21

3.2 Preprocessing and Instrumentation . . . . . . . . . . . . . . . 23

3.2.1 Concrete Trace Recording . . . . . . . . . . . . . . . . . 23

3.2.2 RTL Translation and Analysis . . . . . . . . . . . . . . . 24

3.3 RTL Concolic Execution . . . . . . . . . . . . . . . . . . . 27 
3.3.1 Two-Pass Simulation . . . . . . . . . . . . . . . . 27

3.3.2 Dynamic discovery and mutation of Activated Guards . . . . . . . . . 29

3.3.3 Iterative Bounded Explorations . . . . . . . . . . . . . . . 31

3.4 Experimentation and Results .................. 31

3.4.1 Experiment Setup . . . . . . . . . . . . . . . . . 32

3.4.2 Branch Coverage . . . . . . . . . . . . . . . . . . 33

3.4.3 Test Generation Runtime . . . . . . . . . . . . . . . 34

4 Test Generation using Factored Explorations 35

4.1 Test Decision Tree . . . . . . . . . . . . . . . . . 36

4.2 CORT Framework . . . . . . . . . . . . . . . . . 39

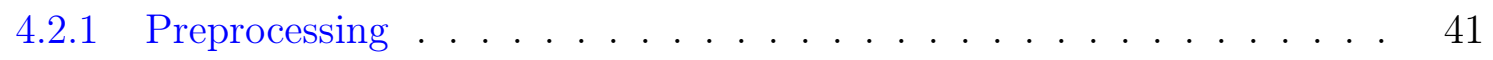

4.2 .2 Concolic Execution Engine . . . . . . . . . . . . . . . 42

4.2 .3 Test Generation . . . . . . . . . . . . . . . . . . . . . . 42

4.2.4 Systematic Exploration . . . . . . . . . . . . . 45

4.3 Experimentation and Results . . . . . . . . . . . . . . 48

4.3.1 Search Strategy . . . . . . . . . . . . . . . . 49

4.3.2 Branch Coverage .................... 50

4.3.3 Effects of Exploration Length . . . . . . . . . . . . . 53

5 Conclusion $\quad 55$

5.1 Limitations and Future Work . . . . . . . . . . . . . . 55

5.2 Conclusion . . . . . . . . . . . . . . . . . 57

$\begin{array}{ll}\text { Bibliography } & 59\end{array}$ 


\section{List of Figures}

2.1 Example for Concolic Execution . . . . . . . . . . . . . . . . . . . 9

2.2 Example HDL design and control flow . . . . . . . . . . . . . . . 12

2.3 Complexity of symbolic analysis . . . . . . . . . . . . . . . . . 13

2.4 Verilog code snippet and its Verilator translated C ++ RTL . . . . . . . 16

3.1 Instrumentation of concrete trace recording in the eval function of the $\mathrm{C}++$ RTL . . . . . . . . . . . . . . . . . . . . . 24

3.2 Example Design-Under-Test . . . . . . . . . . . . . . . . . . . . 25

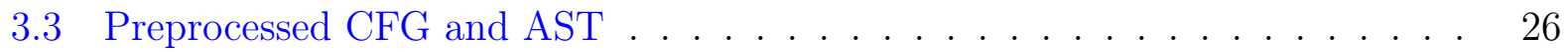

3.4 Concrete evaluation of array index for symbolic expression construction . . . 30

4.1 Test Decision Tree for the example exploration in Section $3.3 \ldots$. . . . . . 38

4.2 CORT Framework . . . . . . . . . . . . . . . . . 40

4.3 Branch Transition Graph . . . . . . . . . . . . . . . . . . . . . 41

4.4 New exploration at $n 3 \ldots \ldots \ldots \ldots$

4.5 Global Test Decision Tree after two explorations . . . . . . . . . . . . . 45

4.6 Tests derived from the TDT and their branch coverage $\ldots \ldots \ldots 46$ 


\section{List of Tables}

3.1 Discovery of Activated Guards using Concolic Execution . . . . . . . . . . . 29

3.2 Benchmark Description . . . . . . . . . . . . . . . . 32

3.3 Comparison of Branch Coverage . . . . . . . . . . . . . . . . 33

3.4 Comparison of Runtime . . . . . . . . . . . . . . . . . . 34

4.1 Benchmark and CORT Strategy . . . . . . . . . . . . . . . 49

4.2 Performance Comparison with Previous Work . . . . . . . . . . . 51

4.3 Extended Performance Comparison . . . . . . . . . . . . . . . 52

4.4 Effects of Exploration Length for b15 . . . . . . . . . . . . . 53 


\section{List of Algorithms}

1 Derive Final Test from the TDT . . . . . . . . . . . . . . . . . . 48 


\section{Chapter 1}

\section{Introduction}

Hardware design validation consumes as much, or more, resources than design development. Rapid test generation for functional metrics at a high level of design abstraction such as the Register Transfer Level (RTL), can aid validation effort. Commonly used functional metrics are akin to their software testing counterparts, i.e., statement coverage, branch coverage, path coverage and assertion coverage [3]. Tests for functional metrics at the RTL help catch design flaws earlier. Considering the complexity of practical hardware design, the go-to standard approach of constrained random test generation is proving to be unsuitable for achieving high functional coverage. Random testing, as popularly used, does not utilize the available high-level functional design information available at this abstraction level. To bridge the gap, designers manually guide directed test generation effort. It is crucial that this task is automated to decrease the overall human effort and redirect it for suitable goals.

Directed test generation by evaluating the design under formal methods can theoretically reach all system states in the design. However, in reality, these techniques do not scale well due to the size and complexity of modern designs. Though it is attractive to use formal techniques such as symbolic execution for the task of automation, it is impractical with 
current technology. Traditionally, symbolic evaluation of RTL [1] is statically performed by unrolling the design for several cycles. Considering that the entire hardware RTL needs to be evaluated per cycle of unrolling, the number of feasible paths that need to be considered grow exponentially (the path explosion ceiling [11]).

This problem has been assuaged to a great extent with the help of Software Testing inspired concolic execution based semi-formal techniques [17, 25, 32], where the expensive task of symbolic evaluation is reduced by restricting it to concrete execution traces stimulated by the random real input. In Software Testing, Godefroid et al. [19] introduced the concept of high performance directed automated random testing (DART) by engaging classical symbolic execution over the concrete execution path derived by testing the software with real (concrete) inputs. Based on this concept, Sen et al. [13] built an efficient C-language unittest generator, CUTE, and coined the term, Concolic Execution (a portmanteau of concrete and symbolic). Current semi-formal concolic execution based RTL testing methodologies score well on their intended functional metric with a minimal number of test vectors but incur a long non-negligible runtime. Furthermore, the search spaces of hybrid concrete and symbolic simulation techniques such as [17] and [25] are bounded to a few tens of cycles due to computational complexity, albeit far superior to traditional static symbolic execution. On the other hand, stochastic or heuristically guided search-based techniques [20,14] are often faster than semi-formal techniques and have the potential to explore a larger search space, but usually, end up producing relatively larger tests to meet similar goals.

\subsection{Contributions of this Thesis}

In this thesis, we introduce CORT (Concolic RTL Test Generator) which aims to generate tests that maximize branch coverage at the RTL of the design-under-test using the least 
number of input vectors. Moreover, CORT was designed with the goal of being able to generate tests as fast as stochastic search-based techniques despite being in the class of RTL semi-formal test generation methods. The expensive costs of hybrid concrete and symbolic evaluation is offset by dynamically analyzing concretely executed statements to restrict the computational costs to segments of the RTL that have been stimulated by the primary input. Furthermore, we highlight the Test Decision Tree, a new perspective in the field of RTL testing where we abstract and record the test generation process in terms of stimuli and control-flow response of the system. Fundamentally, our test generation strategy focuses on systematically growing the Test Decision Tree over several short explorations of highly efficient cycle-by-cycle concolic execution.

CORT has successfully generated high-branch coverage tests for hardware benchmarks from ITC '99 [8] and IWLS-2005 [4]. Experiments show that our methodology is orders of magnitude faster than previous hybrid concrete and symbolic evaluation based techniques while retaining their advantage of achieving a high branch coverage. The tests generated by CORT are significantly smaller than those generated by stochastic search-based techniques.

The contributions of the thesis are summarized below.

- We introduce a cycle-by-cycle concolic execution methodology, where the symbolic evaluation effort is strictly limited to primary input stimulated statements, and unstimulated values are substituted with concrete values from the trace.

- A novel test representation, the Test Decision Tree is proposed, which specifies the multi-cycle control-flow response of the design under various stimuli. Each of our concolic execution based explorations results in a decision tree. Path explosion and limitation of unrolling are overcome by a systematic stitching of the decision trees of many individual explorations to form a global Test Decision Tree. 
- We present CORT, an efficient factored concolic-execution based automated test generator for RTL. Three selection methods are described which heuristically select the next starting state for each factored exploration. Our approach generates the optimal test that reaches all covered branches with the least number of test vectors using the Test Decision Tree.

The work described in this thesis has been submitted to the IEEE International Test Conference (ITC '17), and is currently under review as, S. Pinto and M. S. Hsiao, "RTL Functional Test Generation using Factored Concolic Execution."

\subsection{Thesis Organization}

The remainder of this thesis is structured as follows.

Chapter 2 describes fundamental concepts of hybrid concrete and symbolic evaluation of RTL for the purpose of test generation and related work based on it. The chapter also describes their limitations and methods employed to tackle them.

In Chapter 3, we introduce our novel cycle-by-cycle concolic execution methodology. To demonstrate its performance and efficiency, it is used in a bounded test generation framework similar to previous concolic techniques.

Chapter 4 introduces and describes the CORT framework in illustrative detail. The concept of the Test Decision Tree and its role in the overall factored exploration based test generation process is also exemplified. CORT is benchmarked, and the results are highlighted against previous works.

We conclude this thesis in Chapter 5, describing the current limitations of our methodology and proposals for future work. 


\section{Chapter 2}

\section{Background}

In this chapter, we explain fundamental concepts and terminology that forms our work. This chapter also surveys and contrasts previous work in the field of test generation at the Register Transfer Level (RTL) using concolic execution and how it relates to the work done in this thesis.

\subsection{Automated Functional Test Generation}

Hardware design validation is engaged with the goal of ensuring that the design conforms to its specifications and architecture and that it does not display any behavior inimical to its functional performance. Considering the current scale and complexity of hardware designs, we rely on Hardware Descriptive Languages (HDL) like Verilog and VHDL to abstract the design in terms of its behavioral response and structural components. The task of hardware design is growing analogous to software development. Similar to software development, with the ease of development, it also brings the risk of introducing design errors at the higher abstraction layer, possibly due to direct human error or incorrect interpretation of language 
constructs. The RTL provides high-level behavioral information for directed test generation that can offer a better chance of solving problems like state justification, a primary task in gate-level sequential ATPG (Automated Test Pattern Generation) [30].

At the RTL, functional metrics like statement coverage, branch coverage, toggle coverage or assertion coverage helps with exercising basic structural integrity and measuring behavioral accuracy at that design stage. These functional metrics [3] are derivatives of their equivalent in software development. Without a doubt, automation of test generation effort aimed at maximizing functional metrics or targeted tests that invoke a certain a behavioral response from the design would aid design validation earlier in the design cycle [13]. This not only helps avoid errors that could compound if not caught early but would reduce the overall design cycle time, where hardware design validation currently consumes more time and resources than development.

However, much of the RTL test generation effort is still relegated to constrained or directed random testing. Due to the principal differences between HDL and software languages, the proven techniques from software testing cannot be directly applied to hardware. The HDL usually describes the response of the hardware in the most basic forms of control systems such as finite state machines. The entire HDL is evaluated per cycle per clock in a clocked hardware design. Registers and memory elements hold the state of the system and the next state is decided based on the current state and primary input. Such state registers would be identical to static variables in certain software languages (e.g. $\mathrm{C}++$ ), the use of which is discouraged in practical software development. To adopt directed search-based software testing techniques like [26], [12], and [24], the entire HDL would need to be unrolled per cycle of evaluation, and input domain would grow impractically with the length of the test.

The popular constraint-based formal technique of static symbolic execution [1] or Bounded Model Checking [7, 2] would require the unrolling of the RTL design and evaluation of every 
statement per cycle. Though it is entirely possible to reach every state in the RTL design theoretically, such an exercise quickly proves to be computationally impractical and inviable for real-world designs. In contrast, search-based techniques are oblivious to the behavioral specification of the RTL and rely more on the brute-force random search of the state space, albeit constrained or directed with the help of search optimization strategies. Search-based techniques like [10] and STRATEGATE [21, 22, 6] have successfully employed Genetic Algorithms (GA), a widely popular optimization technique to maximize the state space during explorations for sequential ATPG. Due to its focused approach, STRATEGATE achieved a higher fault coverage due to a greater success at state justification than previous deterministic or simulation-based techniques. Corno et al. [10] used instrumented RTL over a commercial simulator to optimized tests geared towards high statement coverage. Search-based techniques like $[20,14]$ rely on heuristics measured over optimizing branch coverage among their candidate exploratory tests. Undoubtedly, search-based techniques are more popular due to their simplicity of implementation as they often require minimal instrumentation and can operate without severe knowledge of the underlying design, unlike constraint-based techniques. In Software Testing terms, search-based techniques would usually fall under black-box or gray-box testing strategies, while constraint-based testing would be considered as a white-box strategy.

In this thesis, we focus on hybrid or semi-formal techniques that combine the salient advantages of constraint-based and search-based techniques that have been introduced in the field of RTL test generation. 


\subsection{Concolic Execution}

Concolic execution is a hybrid testing technique that combines the classic formal symbolic execution with concrete execution or simulation (in the case of hardware). Godefroid et al. first introduced the technique in his implementation of DART [19], a directed software test generator based on concolic execution. The term concolic execution, a portmanteau of concrete and symbolic execution was coined by Sen et al. [13] in their work on CUTE, a concolic unit test engine for $\mathrm{C}$ language. Their work extended DART for data structures. Symbolic execution is a formal method where the program or design under test is solved with the help of a Satisfiability Modulo Theory (SMT) solver, in terms of its evaluation as symbolic constraint logic. However, in concolic execution, the symbolic execution effort is restricted to the dynamic concrete analysis of the system, rather than static analysis, in order to divert the control flow of the system along a path different than the one concretely executed. The primary focus of concolic execution based testing is finding faults or bugs in the functional behavior of the system under test.

With the advent of powerful SMT solvers like Z3 [34] and Yices2 [35], it has become easier to construct full-featured symbolic interpreters for modern programming languages. Traditionally, in symbolic execution, tests are generated by building and solving a path constraint for a target path in the control flow of the system. All possible paths are investigated in a Depth-First-Search or wavefront manner, without any prior cognizance of feasibility. In concolic execution, the system is first executed with a real or concrete input to obtain a concrete execution trace. The symbolic path constraint is constructed over the statements along that concrete trace. For each branching statement or guards, the path constraint denoting it is negated and solved with an SMT solver to generate the test that certainly guides the control flow along a path that was not concretely executed. The actual superiority of this method is apparent in its ability to restrict symbolic evaluation to statements that have been 
influenced by the input, by only using the symbols for primary input. This reduces the overall computational effort, as fewer symbolic constraints need to be constructed and smaller logical constraints need to be solved, for the same goal as traditional symbolic execution. Nonetheless, concolic execution explores a tree of possible execution paths in a depth-first search manner, and requires being bounded.

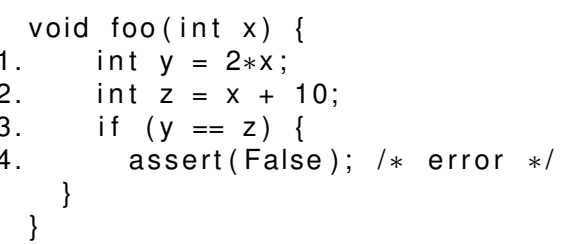

(a) Source Code

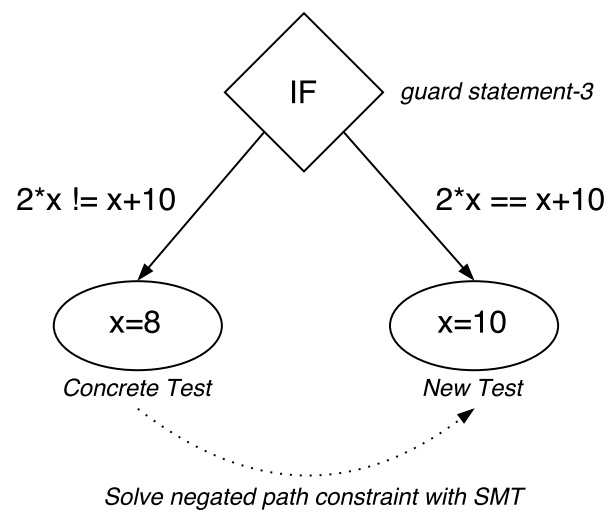

(b) Control Flow Response

Figure 2.1: Example for Concolic Execution

Concolic execution is illustrated over the example software code in Fig. 2.1a. In function foo, the target statement 4 can only be reached when the input integer $x$ holds the specific value of 10 . The test begins with an arbitrary value of $x$, say $x=8$. The concrete execution of foo will result in $y=16$ and $z=18$ in assignment statements 1 and 2 respectively. This leads to guard statement 3 being evaluated to False, and thus taking the else branch. The input is treated as a symbol and the path constraint for the execution is construction for the elsebranch as, $2 * x \neq x+10$. Thus, to divert the control flow along the then branch, input that negates this path condition needs to be applied. The negated conditioned, $2 * x=x+10$, is solved using an SMT solver for $x=10$. On the next run of foo, the input $x=10$ is applied in order to reach the target assertion in statement 4. The control flow response of the function is shown in Fig. 2.1b. The tests are generated corresponding to the leaf nodes in the response tree. 


\subsection{Scaling through Factored Exploration}

A distinct difference between hardware and software with respect to its formal techniques like Bounded Model Checking (BMC) and Symbolic Execution is in the abstract functional description of the systems (HDL for hardware, and programming languages for software). The HDL source of the hardware consists of both concurrent and procedural statements and code blocks. The hardware is described in terms of its response to primary-input stimuli and its stored system states in memory elements such as registers and memory arrays. For sequential hardware, the HDL is usually synchronized to one or more clocks. Per clock cycle, not all system states are reachable regardless of primary input. The entire HDL has to be unrolled and evaluated per clock cycle. Any practical test for hardware will consist of a non-negligible number of cycles. It has been previously mentioned in this thesis that such large unrolling makes symbolic execution unrealistic, even under the assumption that a full featured symbolic interpreter is present for the HDL design under test. Nonetheless, to overcome this clear limitation of formal methods under deterministic computing paradigms, clever techniques like inductive reasoning and variable ordering strategies have been successfully implemented. In [18], SAT-based (Boolean Satisfiability) BMC was scaled through variable ordering that is learned during incremental unrolling, and efficient BMC-specific ordering tactics implemented were in the core SAT solver. With the help of signal correlations, implications were deduced across multiple cycles of unrolling in [27]. This allowed their SAT-based BMC to scale phenomenally beyond previous work at that time, as it could reduce the overall search space.

The primary input is engaged for every cycle in the HDL, and every primary-input signal requires being cycle-annotated to be evaluated symbolically. The degree of unrolling decides the complexity of the symbolic execution. Such a formal evaluation of the HDL can be understood as having static or state variables in software functions that are engaged in an 
infinite loop [24]. This property is unique to hardware as software testing does not require unrolling of cycles as in hardware and the concrete path under evaluation begins at distinct start and end system state. In traditional hardware concolic execution [17], all symbolic evaluation along a concrete simulation trace begins at predefined state (say, reset) prior to which no signal has been stimulated by the primary input. At this starting state, all signals are either assigned initial constant values or some function of the primary input. For each cycle hence, it is easy to detect signals influenced or stimulated by cycle-annotated primary input by following the use-definition [26] chain. This is akin to software testing of a function where no function-scoped variable has a deterministic value before execution. Despite having the property to control the complexity of symbolic execution by limiting the unrolling, the effort necessarily begins at a fixed starting state. Thus, a maximum bound for cycles that can be concolically simulated for test generation from the starting state is unfortunately conditioned on computational power and time at disposal.

However, with appropriate instrumentation, concolic execution for HDL can begin at any system state. This is enabled by having access to values of memory elements through simulator instrumentation. Say, a new exploration needs to begin a system state that is 1000 cycles away from the predefined reset state, then, after simulation of that base test the values of all memory elements form the initial values for symbolic execution (constrained to primary input stimulated statements) of the new test. Though this constrains the input search domain to the cycles of simulation; it allows for path constraints to be constructed in a search space previously computationally unreachable. The search space can be factored to computationally feasible exploration sizes (test length) using concolic execution. This allows for piecewise test generation similar to iterative or generational search-based techniques, a feature previously not conceived for hardware constraint-based techniques. In software concolic execution, without heavy instrumentation, a large functional (say, 1000-line) concrete 
path cannot be split into parts and individually symbolically analyzed. In hardware, we can split a long test (say, 1000 cycles) into incremental parts, reading the system state before and after each part.

Consider the simple Verilog HDL example in Fig. 2.2b, where a register count has to be incremented before having an opportunity to reach the target statement. This would require at least 16 cycles, i.e. 15 cycles of incrementing count and 1 cycle for initialization (reset state). Aside from the functional clock and reset input, key is the primary input that allows us to guide the control flow towards the target at the right moment. The control flow graph of the example design is shown in Fig. 2.2c, where we observe 4 unique paths that can be executed.

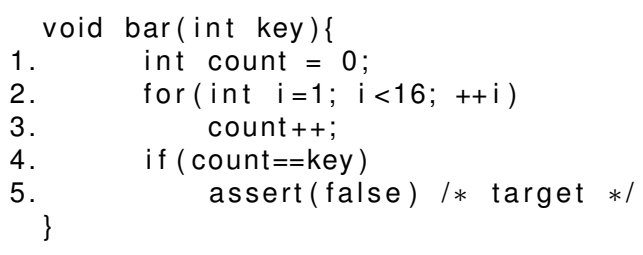

(a) Software equivalent

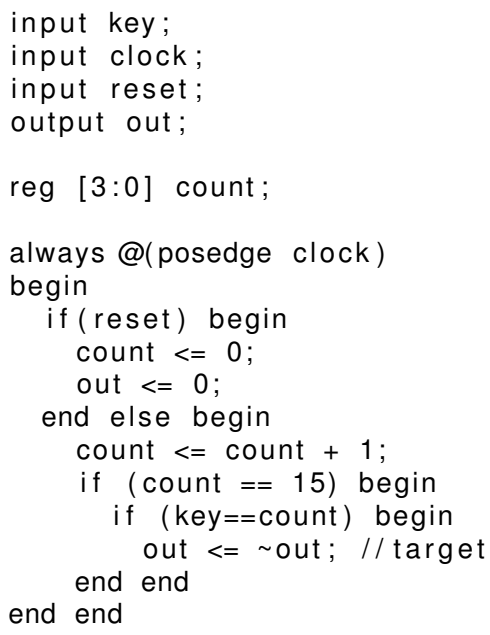

(b) Verilog HDL design

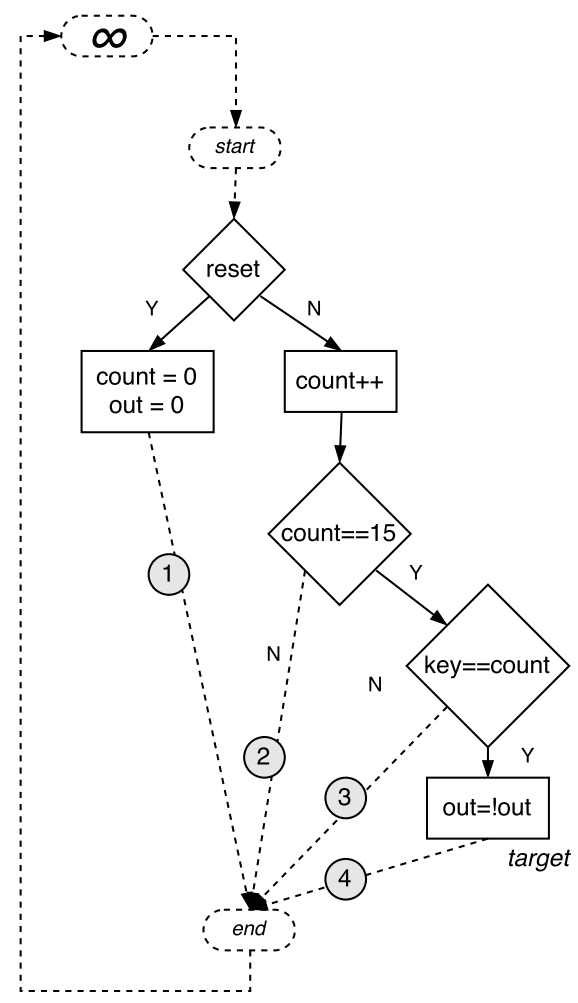

(c) Control Flow

Figure 2.2: Example HDL design and control flow 
In the first necessary reset cycle, path-1 is taken. Pursuant of bounded concolic execution (with concrete stimuli of $k=0$ ), we would have to begin analysis at this state which provides constant initial values for variables participating in the execution. For the next 15 cycles, path-2 is executed, and the path-constraint grows accordingly. In the $16^{\text {th }}$ cycle, the concrete input $k e y=0$ leads the execution away from the target, along path-3. Overall, the relevant symbolic path constraint for these 16 cycles is described in Fig. 2.3a. The large path constraint associated with the target requires the incremental symbolic computation of count (assuming for the sake of the example that no advanced symbolic execution optimization techniques are being used). Without knowledge of initial values of count this evaluation would not be possible. This situation where the evaluation is forced to begin at predetermined start state is akin to the Input-Output testing of functions in Software Testing [26], where all variables are scoped within the function and evaluation begins at the start of the function block. The evaluation cannot begin at a point within that function, as we would not know the values of variables altered before that point (without extensive instrumentation to access the concrete environment). Looking at the software equivalent in Fig $2.2 \mathrm{a}$, we see that the formal analysis cannot simply begin at statement-4. The value of count needs to be symbolically determined over the statements 2-3 from its initial definition in statement-1.

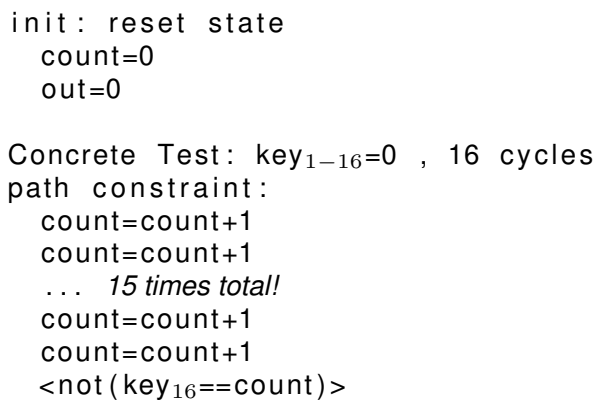

(a) 16-cycle path constraint

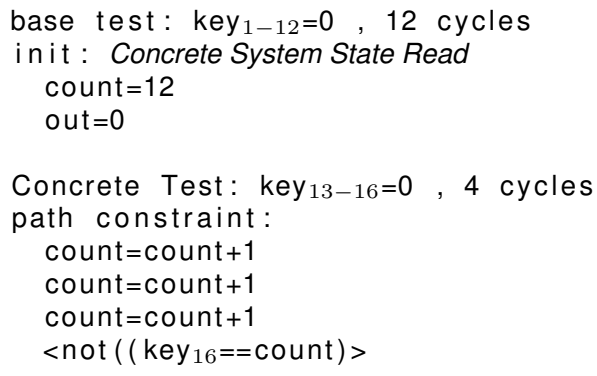

(b) Path constraint for segment-4

Figure 2.3: Complexity of symbolic analysis 
Assume that instrumentation to access any signal in the simulated design is available. Being able to concretely read the system state at any cycle would not only allow the test to be at a state away from the reset state, but also enable the ability to factor the test. Let us assume that the 16-cycle test is factored into four parts, each of length 4 with the same concrete stimuli of $k e y=0$ throughout. Consider the first segment (cycles 1-4) which concretely simulates the first four cycles of the same execution as in Fig. 2.3a. However, no path constraint would need to be built in this factored segment as no statement was influenced by input (implying that control flow in the segment cannot be changed as no stimulus is consumed). The test so far forms the base test for the next segment, i.e. the concrete stimuli of segment-1 is applied before concolic execution of segment-2. Once, the base test is simulated, the system state is concretely read to determine the initial values for concolic execution in the current segment.

The case for the segment-2 (cycles 5-8) and segment-3 (cycles 9-12) would be the same as segment-1, as the system continues to execute through path-2. For the fourth segment (cycles 13-16), the first three segments form a cumulative base test of 12 cycles which would lead to the system state consisting of count $=12$ and out $=0$. This system state is read from memory elements (registers and arrays) and provide initial values for the formal analysis distant from the reset state. The concrete stimuli for segment- 4 are four cycles of $k e y=0$ leading to a concrete trace that goes through path-3 in cycle-16. The path constraint for this factored segment is shown in Fig. 2.3b. The size and computational complexity of this 4-cycle path constraint are smaller than the 16-cycle path constraint. The cyclic execution of hardware forms a natural boundary for factoring and analysis where the system state can be read between cycles (or segments of cycles).

While it true that a larger path constraint has a greater visibility of the effect of primary input on the control flow, it is also expensive. Factoring the exploration into smaller tests 
and obtaining the initial values of signals through instrumentation, each time bringing the system away from the initial state, is the compromise we adopt to scale our concolic execution methodology.

\subsection{Verilator and RTL Instrumentation}

Verilator [36] is an open source transpiler (source-to-source compiler) that converters Verilog HDL into a high-performance $\mathrm{C}++$ behavioral simulator. Given synthesizable Verilog, Verilator outputs a $\mathrm{C}++$ class representing a cycle-accurate functional model of the RTL. All signals (IO and internal) of the top hierarchical component of the design are directly accessible as public data members of this class. The class also provides a public function, eval, which simulates the model's behavioral response based on the current values of its public data members.

Furthermore, Verilator automatically instruments the source code for branch coverage measurement. Counters are placed under decision branches which increment when the control flow executes through them. Simulating the model for a single cycle can be understood as assigning the data input, followed by two calls to eval with complimentary values set for clock input. All Verilog branching logic (case, if/else) are converted to optimized nested if/else chains in the $\mathrm{C}++\mathrm{RTL}$. Verilog arrays are converted to contiguous $\mathrm{C}++$ traditional arrays (non-STL). An example Verilog snippet and its translated C++ RTL is shown in Fig. 2.4. $\mathrm{A} \mathrm{C}++$ class, Vtop forms the top hierarchical component that is structurally equivalent to the top module in the Verilog source. The branch coverage instrumentation, VCoverage is a contiguous array of length equivalent to the number of branches in the behavioral code.

Since every signal of the Verilog design can be accessed or mutated in an object oriented manner as public data members of the top hierarchical class, the simulator that is compiled 


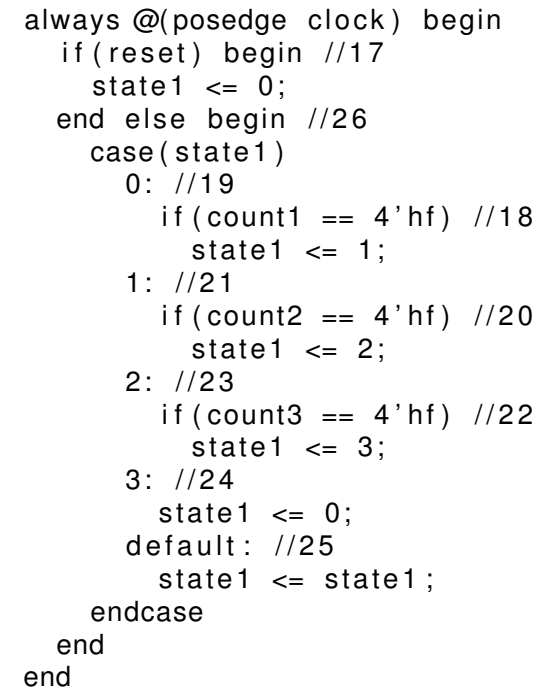

(a) Verilog HDL

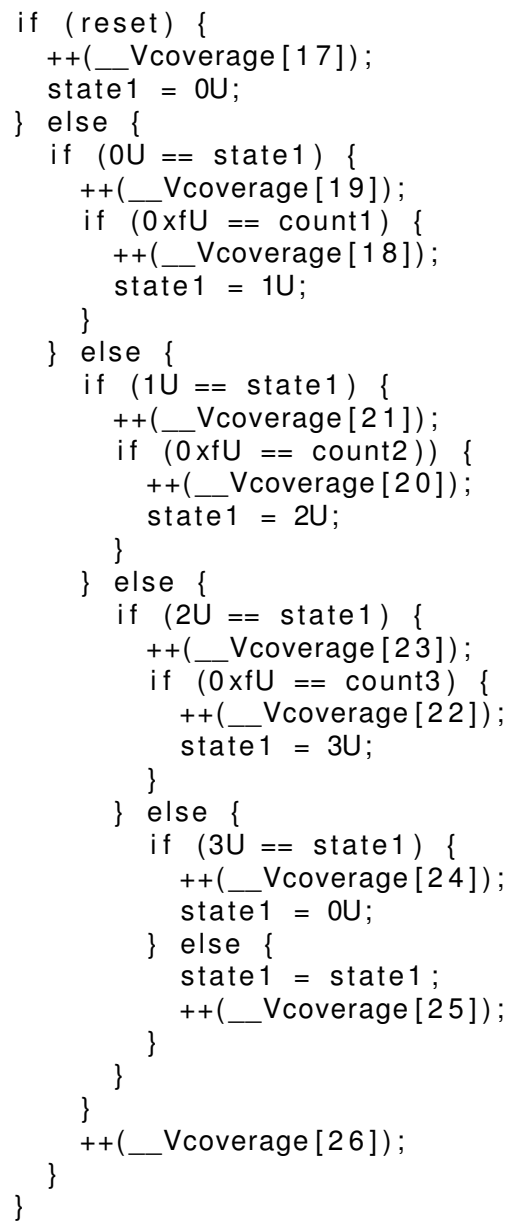

(b) Verilated $\mathrm{C}++\mathrm{RTL}$

Figure 2.4: Verilog code snippet and its Verilator translated C ++ RTL

using the Verilated model $(\mathrm{C}++\mathrm{RTL})$ is explicitly well suited for concolic execution. While building path constraints, used signals that have not been stimulated by primary input can simply be read from the simulator instance and substituted as concrete values. In the traditional symbolic evaluation, the value for unstimulated variables would be evaluated from its last definition. 


\subsection{Related Work}

HYBRO [17] demonstrates viable RTL directed test generation by symbolically executing the concrete trace extracted from the design Control Flow Graph (CFG) [9] during simulation. The RTL is instrumented with branch coverage counters, and the execution path in the CFG is deduced by observing the change in branch counters per simulation cycle. From a fixed starting state, the design is concretely simulated using random vectors. The entire extracted concrete trace is then symbolically evaluated to reveal activated (stimulated by input) guards along that trace. In HYBRO, both cycle-annotated primary input and unstimulated internal signals are considered for the symbolic execution effort. The path constraints for activated guards are negated (mutated) and solved using an SMT solver, in the reversed order of their concrete execution. The solution (in terms of primary input) that satisfies the negated path expressions for the guards is used to form the new test that offers an exploration of a new region in the test. HYBRO improves upon STAR [16] where previously mutated guards would have been retained, resulting in repeated execution of previously explored paths. As a compromise, in subsequent iterations, mutation is not attempted on guards that lead to branches that have been explored within the bounded region. Thus, HYBRO poorly relies on the random concrete stimuli to reach system states that require repeated execution of certain paths (e.g. loops and counters). Moreover, HYBRO does not support HDL array elements or memories which are integral parts of practical designs.

Xiaoke and Mishra [25] also interleaved concrete simulation and symbolic execution, by instrumenting the RTL with statements that printed the concrete execution in terms of syntactic elements. The design is simulated with random stimuli and a trace file representing the concrete execution is output. The resultant trace file is symbolically analyzed to reveal activated statements. In contrast to HYBRO, which symbolically evaluates unstimulated variables used in activated statements, their work could simply substitute them with their 
concrete equivalent (parsed from the print statements in the trace file). [25] also supports symbolic evaluation of memory arrays by treating them as individual index-annotated scalar variables. In a statement where an array variable is used, the index is evaluated as concrete, and the appropriate symbol for the memory index is substituted. Both [17] and [25] generate tests by unrolling the design for a fixed number of cycles from a specific starting state and are limited to the exploration of system states reachable within the unrolled cycles.

PACOST [32, 33], a target oriented RTL test generator has shown success in reaching deep hard-to-reach states by using factored explorations adjunct to interleaved concrete and symbolic simulation. Both the single-cycle and the multi-cycle path constraint variant of PACOST relies on systematically building the test over smaller explorations. In an exploration, the state space is explored in a similar manner to [17], where the guards are mutated in the symbolic path-constraint constructed over the concrete execution. Successive explorations begin at a heuristically determined starting state discovered in the current exploration. A selection of a starting state is determined from state distances derived from an abstract model of the RTL [29]. However, the construction of path constraints in each exploration is limited to variables that can trace their use-definition chain to an input variable within that exploration. If any variable in a guard path expression cannot be defined solely in terms of primary input, then no mutation effort is performed on that guard. In contrast to PACOST, our factored explorations are guided by heuristic metrics derived from the overall coverage status of the test, and our analysis is not restricted to use-definition chains wholly contained within the exploration. Furthermore, in PACOST, abstraction of the RTL leads to loss of information and the calculation of abstract state distances is limited by their BMC-based preprocessing.

Guiding the direction of test generation by dynamically calculating branch coverage based heuristics is described in BEACON [20]. It is a search-based testing technique which uses a 
hybrid Ant Colony Optimization and evolutionary algorithm to maximize branch coverage in the RTL design under test. The Verilog RTL source is translated to a functional $\mathrm{C}++$ model and is instrumented for branch coverage measurement. The underlying search heuristics are updated at each iteration during simulation. BEACON is able to reach hard-to-reach states in a runtime that is orders of magnitude shorter than HYBRO. This is expected as it does not incur the cost of expensive SMT solver operations. To further improve its performance, in [15], BEACON was extended with a feature to periodically perform a local state space search using an SMT-based Bounded Model Checker. This allows the search-based algorithm to detect branches that could not have been found by a stochastic search alone. 


\section{Chapter 3}

\section{Cycle-by-Cycle Concolic Execution of RTL}

In this chapter, we introduce a novel concolic execution methodology geared towards rapidly generating tests at the Register Transfer Level (RTL). The goal of this methodology is to generate a multi-cycle sequential test that maximizes branch coverage, in the shortest amount of time. The following is a brief overview of our hybrid concrete and symbolic simulation test generation method. The overall test is iteratively built over several explorations using randomly generated concrete input vectors (concrete stimuli), from a predetermined starting system state (say, system reset), bounded to a fixed number of simulation cycles. For each exploration, we perform cycle-by-cycle concolic simulation where symbolic expressions are only constructed for primary input stimulated (activated) statements in the concrete execution trace. Not all statements or variables are data-dependent on primary input. Such statements can be abstracted away, and the unstimulated variables defined by them can be substituted for their concrete values (read from the simulator) if they are eventually used in

other activated statements. As a result, the overall symbolic evaluation effort is significantly 
reduced.

The objective of concolic simulation is to dynamically reveal activated control points (guard statements) in the trace. The symbolic expressions for activated guards are mutated (negated) using a formal Satisfiability Modulo Theory (SMT) solver, to generate mutation stimuli. Altering the concrete test with the specific mutation stimuli generates the test that reaches branches that were not executed during the current exploration. For the purpose of experimentation, a bounded test generator based on this concolic execution methodology is built. In each exploration, the test for each discovered branch is recorded. The overall test is the optimal concatenation of tests selected from pool of all tests recorded so far, such that all discovered branches are covered.

The cycle-by-cycle concolic execution methodology proposed in this thesis offers a significant reduction in functional test generation time compared to previous work [17, 25] based on similar hybrid concrete and symbolic evaluation.

The rest of the chapter is organized as follows. Terminology for key components of our concolic execution algorithm is described in Section 3.1. In Section 3.2 details the preprocessing and instrumentation of our RTL simulation framework. Our cycle-by-cycle concolic execution methodology is described in great detail over an example in Section 3.3. Lastly, our method is tested against previous work in Section 3.4.

\subsection{Terminology}

A concrete path or trace is defined as a sequence of statements (assignments and guards) executed upon simulating the RTL source code (for one or more cycles) with concrete input stimuli. However, unlike previous work, we do not construct a path constraint which 
represents concrete trace as the stack of symbolic assignments and guards. Rather, we perform cycle-by-cycle concolic evaluation and maintain an Activation Table which stores the symbolic definition of activated variables defined in assignment statements. In our work, concolic execution requires symbolically executing the concrete path by only using symbols for inputs. In each cycle, a symbolic expression is constructed for a statement if it is found to be activated, with appropriate substitutions for each variable used. For primary inputs, the cycle annotated input symbol representing them are used. An activated variable is substituted for its defining symbolic expression fetched from the Activation Table. Values of unstimulated variables are said to be concrete, and are obtained (read) from the simulator during simulation (between cycles).

Along a concrete trace, guards that use activated variables or primary inputs are termed activated guards. For each activated guard, a boolean symbolic expression representing its predicate and the concrete branch taken is constructed. A guard expression is a function of input stimuli. To divert the control flow, we attempt to mutate the activated guards. An activated guard that can be mutated is called a mutable guard and the stimuli that satisfy the mutation is referred to as the mutation stimuli. The constraint stack for each activated guard includes its negated expression and the concrete expressions for the intersecting mutable guards found before it, in this trace. The intersection of guards is defined as having cycleannotated input stimuli in common, and we consider this during the mutation effort to ensure that the mutation stimuli of a latter guard do not mutate the concrete execution of a former. The constraint stack is solved using an SMT solver. If the constraint stack could not be solved, then the activated guard is ignored henceforth in the analysis of this trace.

Comparable to [25], our work can build symbolic expressions for statements involving array accesses (e.g. memory, queues, FIFO) where every element in the array is treated as a scalar variable annotated by its index. This means that the array index would be read as 
concrete. Thus, upon encountering activated variables being used as array access indexes, we deactivate them and consider their input stimuli to be immutable stimuli. No symbols are created for immutable stimuli, and their concrete values are substituted wherever they are used. In a similar note, our concolic execution methodology considers clocking and reset inputs as immutable stimuli, and always treats them as concrete values.

\subsection{Preprocessing and Instrumentation}

This section describes the preprocessing tasks that occur before the real test generation effort. The RTL is instrumented for both branch coverage and trace recording, the latter being an enhancement to Verilator introduced as a part of our work.

\subsubsection{Concrete Trace Recording}

Recording the concrete trace traversed in the design in each cycle is a fundamental operation in our work. To this end, Verilator [36] (introduced in Section 2.4) compiler source code is modified to allow for instrumentation of concrete trace recording during functional simulation. The top hierarchal class which simulates the design, Vtop is extended with a $\mathrm{C}++$ integer vector named trace. In the eval function, each branch coverage increment statement is followed by a command to concatenate the branch ID into the trace (Fig. 3.1). The trace data structure is cleared before a cycle and read at the end of a cycle. During a call to eval function, when the execution flows through a path, it is recorded in the trace as unique sequence of branches. The accumulated sequence of integer branch IDs denotes the concrete trace and is accessed as a public data member of the top simulator class. 


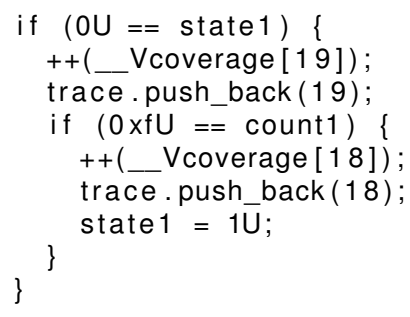

Figure 3.1: Instrumentation of concrete trace recording in the eval function of the $\mathrm{C}++$ RTL

\subsubsection{RTL Translation and Analysis}

The task of preprocessing begins with the translation of the Verilog source of the design into $\mathrm{C}++$ using Verilator. Branch coverage measurement (in-built Verilator feature) and execution trace recording are instrumented during the translation. The coverage status for the simulation is the value in each instrumented branch coverage counter. The Verilog source for the design used as the motivating example in the following section is presented in Fig. 3.2a. The instrumented $\mathrm{C}++$ code (with simplified variable names for the purpose of illustration) representing its cycle-accurate behavior is displayed in Fig. 3.2b.

The Abstract Syntax Tree (AST) for the model's eval (behavioral model) function is parsed from the Verilated design source code and is transformed into a Control Flow Graph (CFG) [9]. The AST structure for each statement helps with analysis and construction of symbolic expressions. A single-cycle execution trace of branch IDs corresponds to a unique path in the CFG describing both the assignment statements and the concrete execution of guard statements. In the RTL, by construct, certain branches might be unreachable, i.e. there exists no test that can guide the control flow through that segment of the code. Verilog default segments in case statements are the usual examples for these cases. Identification of such branches helps narrow down the goal space (branch coverage). In this work, we use signal domain analysis as introduced in [28] across the AST to identify predicates (branch conditions) that cannot be satisfied under any assignment of the predicate variables. How- 


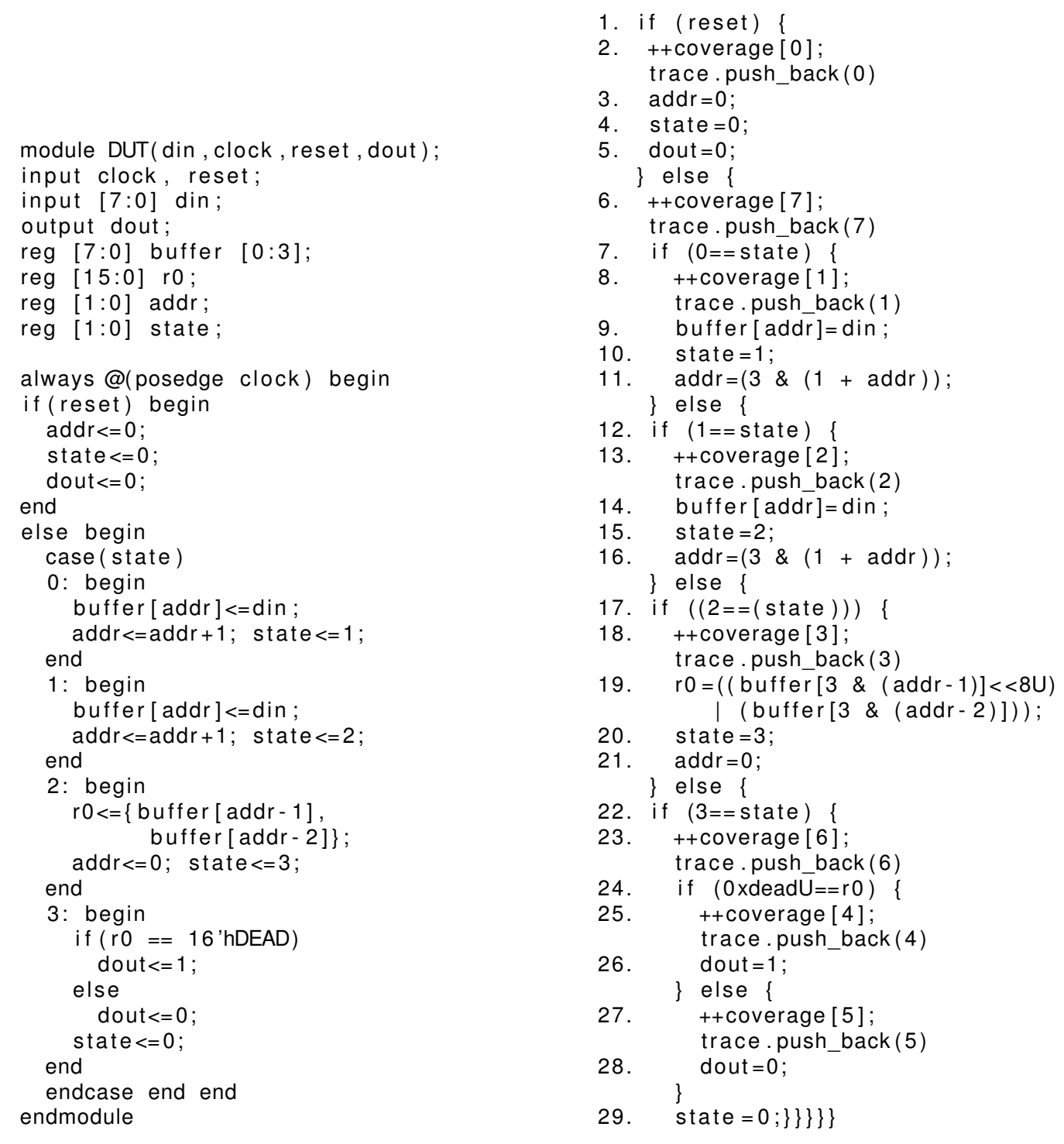

(a) Verilog RTL

(b) Instrumented $\mathrm{C}++\mathrm{RTL}$

Figure 3.2: Example Design-Under-Test

ever, we use a non-formal approach [31] by restricting the signal domain range to predicate control signals that are only assigned constant values across all statements that define them. In simple terms, given that a predicate variable can take a value from a finite set of values (parsed from the AST), satisfiability is checked in predicates that use that signal. Branch expressions which cannot be satisfied are said to be unreachable branches. 
Lastly, a simple harness that instantiates the $\mathrm{C}++$ model and provides generic accessor functions to its public members (signal variables, coverage counters, trace, and eval) is generated. The harness is compiled along with the model to obtain the design simulator. The CFG for the example design is shown in Fig. 3.3a. The AST forming statement-19 is portrayed in Fig. 3.3b, highlighting a complex expression that utilizes arrays.

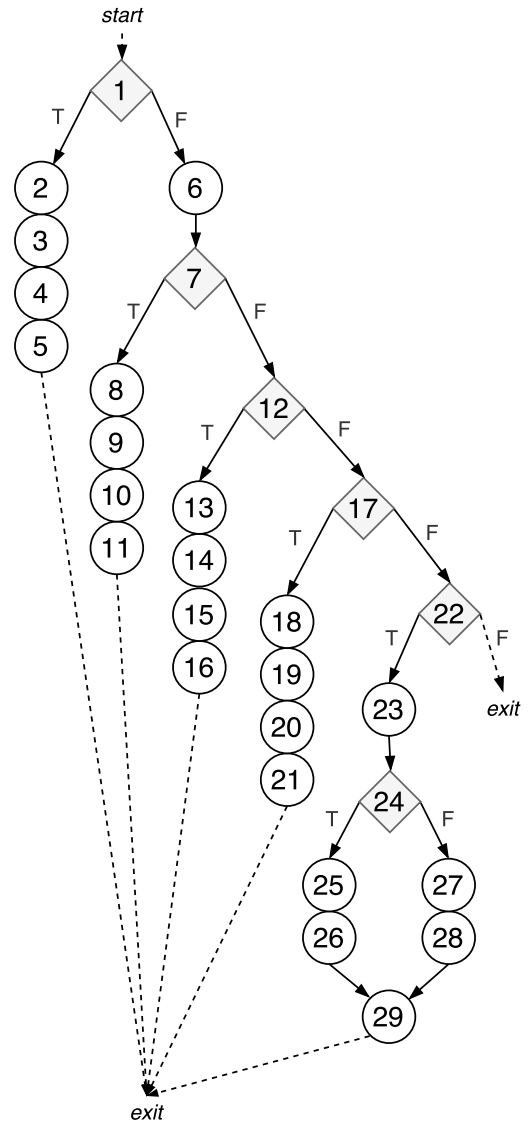

(a) Control Flow Graph

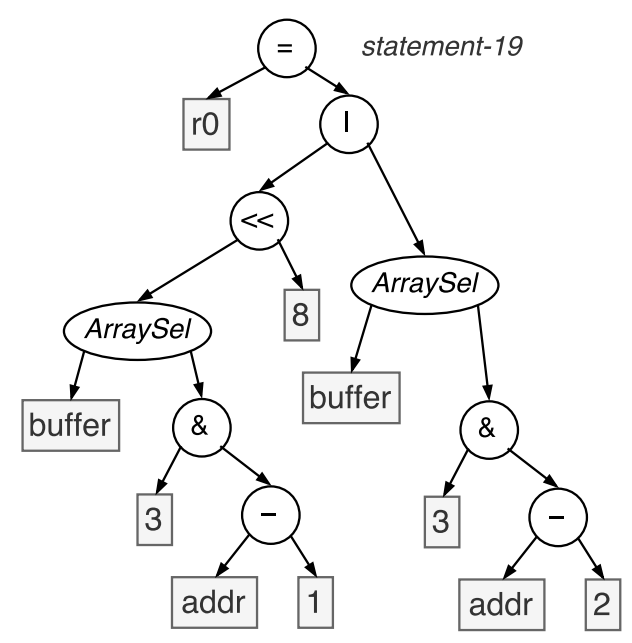

(b) AST for statement-19

Figure 3.3: Preprocessed CFG and AST 


\subsection{RTL Concolic Execution}

In this section, our concolic execution methodology is illustrated over the example design in Fig. 3.2. The following subsections demonstrate a concolic exploration of the design and the cycle-by-cycle simulation and analysis is annotated in Table 3.1. For this example exploration, assume a random concrete input of five cycles (cycles 1-to-5 in column 2).

Before simulating the model with the concrete input, user-provided initialization vectors are applied to the model. In this case, that would be a single cycle of primary input reset set to high, during cycle-0, beyond which it is held low. This brings the system to the required starting state. No analysis is performed over the initialization cycles.

\subsubsection{Two-Pass Simulation}

Though we have access to concrete values of all signals by simply reading them directly from the simulator (instead of parsing simulation print-outputs as in [25]), the values can only be read between clock pulses, not during it. Therefore, we require at least two passes through the same concrete stimuli to have valid requisite pre-cycle or post-cycle concrete reads. Each simulation pass begins by applying the initialization sequence. The Activation Table is only required for the second pass and starts off empty. Firstly, as the functional simulation of the $\mathrm{C}++$ translated RTL is at least five times faster than native Verilog simulation, such an overhead is acceptable. Secondly, being able to read and substitute concrete values for unstimulated variables avoids the redundant effort of symbolically evaluating unstimulated statements, as done in [17]. We take advantage of the fact that the simulator functionally evaluates every statement and variable regardless of stimulation, and that we have easy access to read any variable value during simulation. 
- Pass-1: Concrete Trace Analysis. The design is simulated over the concrete stimuli. The concrete execution trace (column 3) traversed in the model is inferred from the CFG by observing the trace recorded after the cycle. Say, in cycle-1, by noting a trace of $\langle 1,7\rangle$, we can deduce that the execution followed a concrete path of $<1 \mathrm{~F}-6$ 7T-8-9-10-11> where the guards in statements 1 and 7 evaluated to False (else) and True (then) respectively. By analyzing the statements along the per-cycle trace, we mark each used variable as a post-cycle or pre-cycle read, for their particular cycle. A used variable in marked as a post-cycle-read if the variable was first defined (Verilog blocking assignment) and then utilized in the same cycle. Else, it is characterized as a pre-cycle-read. Symbolic analysis of the concrete trace is performed in the second pass.

- Pass-2: Concolic Execution. In this pass, before simulating a clock pulse with concrete stimuli, we first analyze the previously extracted concrete path for the upcoming cycle, to reveal activated statements (stimulated by primary input or an activated variable from the Activation Table). The used variables in each activated statement which were marked as pre-cycle-read for this cycle, are now read from the simulator. Next, the clock pulse is applied. Symbolic expressions (column 5) are constructed for activated statements using its AST representation with appropriate value substitutions for the used variables. Post-cycle concrete reads for unstimulated variables are read from the simulator on demand. The defined activated variable is enlisted in the Activation Table (column 6). The concrete reads in each cycle are shown in column 4. 
Table 3.1: Discovery of Activated Guards using Concolic Execution

\begin{tabular}{|c|c|c|c|c|c|}
\hline Cycle & $\begin{array}{l}\text { Concrete } \\
\text { Stimuli }\end{array}$ & $\begin{array}{l}\text { Concrete } \\
\text { Trace }\end{array}$ & $\begin{array}{l}\text { Concrete } \\
\text { Reads }\end{array}$ & $\begin{array}{c}\text { Statement } \\
\text { Expressions }\end{array}$ & $\begin{array}{c}\text { Activation } \\
\text { Table }\end{array}$ \\
\hline 0 & reset $=1$ & - & - & - & - \\
\hline 1 & $\operatorname{din}=68$ & $\begin{array}{c}\text { 1F-6-7T-8-9-10- } \\
11\end{array}$ & $\operatorname{addr}=0$ & 9: buffer $[0]=\operatorname{din}_{1}$ & buffer_0 : $\operatorname{din}_{1}$ \\
\hline 2 & $\operatorname{din}=178$ & $\begin{array}{l}\text { 1F-6-7F-12T- } \\
13-14-15-16\end{array}$ & $\operatorname{addr}=1$ & 14: buffer $[1]=\operatorname{din}_{2}$ & $\begin{array}{l}\text { buffer_0 : } \operatorname{din}_{1} \\
\text { buffer_1 } \operatorname{din}_{2}\end{array}$ \\
\hline 3 & $\operatorname{din}=75$ & $\begin{array}{c}1 \mathrm{~F}-6-7 \mathrm{~F}-12 \mathrm{~F}- \\
17 \mathrm{~T}- \\
18-19-20-21\end{array}$ & $\operatorname{addr}=2$ & $\begin{array}{c}19: \\
r 0=(\text { buffer }[1] \ll 8) \mid \text { buffer }[0] \\
=\left(\operatorname{din}_{2} \ll 8\right) \mid \operatorname{din}_{1}\end{array}$ & $\begin{array}{c}\text { buffer_0: } \operatorname{din}_{1} \\
\text { buffer_1 : din } \\
r 0:\left(\operatorname{din}_{2} \ll 8\right) \mid \operatorname{din}_{1}\end{array}$ \\
\hline 4 & $\operatorname{din}=232$ & $\begin{array}{l}1 \mathrm{~F}-6-7 \mathrm{~F}-12 \mathrm{~F}- \\
17 \mathrm{~F}- \\
22 \mathrm{~T}-23-24 \mathrm{~F}-27- \\
28-29\end{array}$ & - & $\begin{array}{c}\text { Activated Guard } 24 \mathrm{~F}: \\
\operatorname{not}(57005==r 0) \mapsto \\
\neg\left(57005==\left(\operatorname{din}_{2} \ll 8\right) \mid \operatorname{din}_{1}\right)\end{array}$ & $\begin{array}{c}\text { buffer_o : din }{ }_{1} \\
\text { buffer_1 } 1: \operatorname{din}_{2} \\
r 0:\left(\operatorname{din}_{2} \ll 8\right) \mid \operatorname{din}_{1}\end{array}$ \\
\hline 5 & $\operatorname{din}=22$ & $\begin{array}{c}\text { 1F-6-7F-8-9-10- } \\
11\end{array}$ & $\operatorname{addr}=0$ & 9: buffer $[0]=\operatorname{din}_{5}$ & $\begin{array}{c}\text { buffer_0: } \operatorname{din}_{5} \\
\text { buffer_1 : din } \\
r 0:\left(\operatorname{din}_{2} \ll 8\right) \mid \operatorname{din}_{1}\end{array}$ \\
\hline
\end{tabular}

\subsubsection{Dynamic discovery and mutation of Activated Guards}

For this example, $\operatorname{din}_{i}$ denotes the byte input $\operatorname{din}$ on cycle $i$. In cycle-1, the first cycle of analysis, the assignment statement-9 is found to be activated by the input din. It is seen that this statement involves the definition of an index in the array buffer. We require that the array access index variables themselves are concrete. Thus, the concrete value for $a d d r$ is pre-cycle-read from the simulator and the index is calculated by substituting it in the AST for array index selection (ArraySel AST node). To construct the symbolic expression, the AST is traversed in a depth-first search manner, recursively building expression components. In the LHS of the activated statement, we use $\operatorname{din}_{1}$ for the construction of the symbolic expression defining buffer[0]. Finally, we add the index annotated array variable, buffer_o and its symbolic expression: $\operatorname{din}_{1}$, to the Activation Table. Similarly, in cycle-2, statement- 
14 is found to be stimulated, and the Activation Table is updated with buffer_ 1 with its expression: $\operatorname{din}_{2}$.

During cycle-3, statement-19 is found to be activated as it uses activated variables buffer_o and buffer_1. The symbolic expression for $r 0$ is constructed by substituting values for activated variables from the Activation Table in statement's AST representation, as shown in Fig. 3.4. In cycle-4, statement-24 is the first activated guard to be encountered along the overall concrete path. We see that, with the concrete stimuli, the execution takes the else branch, and thus, the appropriate predicate expression is constructed. Statement-9 is re-activated in cycle-5 and updated in the Activation Table but is not used further in this exploration. While not shown in the example, it must be noted that if an entry in the Activation Table is found to be deactivated (defined wholly by unstimulated variables or constants) while analyzing the concrete path, it is simply removed.

LHS: ro, RHS: buffer[1] $<<8$ I buffer[0]

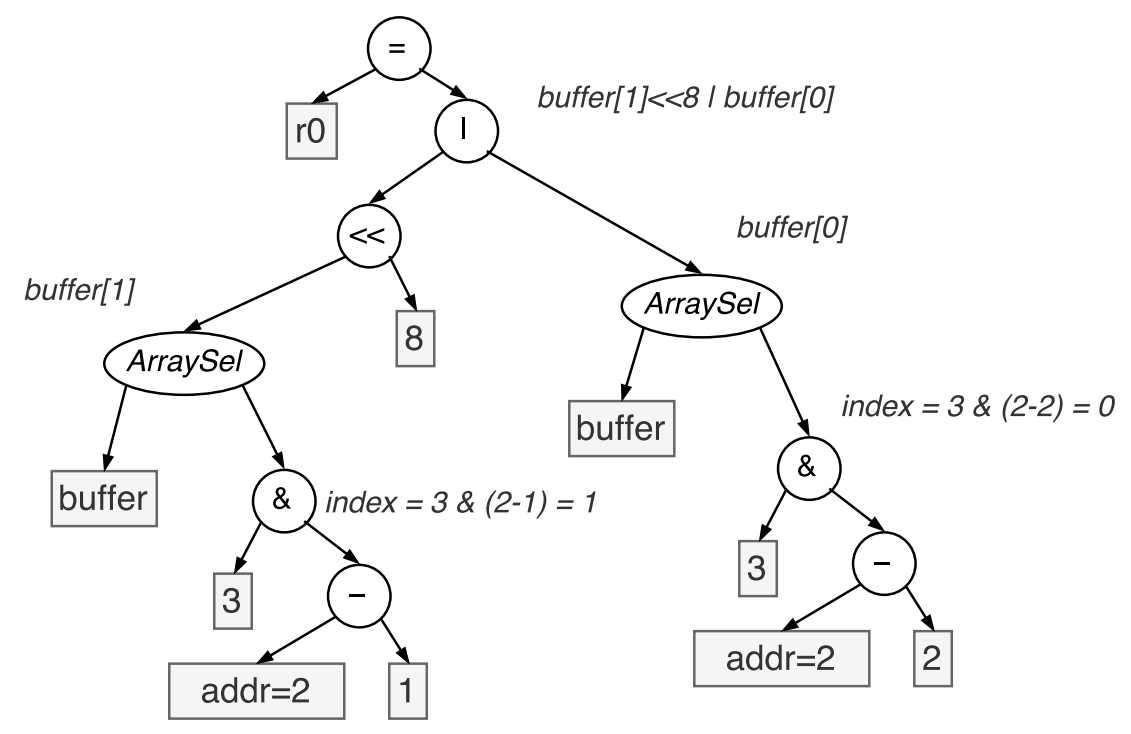

Figure 3.4: Concrete evaluation of array index for symbolic expression construction

Once all the activated guards are discovered, an attempt is made to check if they are mutable. The negated expression for each activated guard is fed to the SMT solver con- 
strained by expressions for mutable guards with which its stimuli intersects. In the current example, the expression representing activated guard-24 on cycle-4, is negated to obtain $\left(57005==\left(\operatorname{din}_{2} \ll 8\right) \mid \operatorname{din}_{1}\right)$. The solver returns $\operatorname{din}_{1}=173$ and $\operatorname{din}_{2}=222$ as mutation stimuli, and guard-24 is found to be a mutable guard in cycle-4.

\subsubsection{Iterative Bounded Explorations}

The test that reaches branch-4 is built by overwriting the current concrete test with the mutation stimuli. The new test is simulated to verify that the control flow reaches the target branch, and recorded as the test for that branch. For the purpose of experimentation and evaluation of our concolic execution methodology against previous work [17, 25], a simplistic bounded test generator is built. The strategy followed is similar to HYBRO [17] where the process of concolic execution is performed over several iterations using random concrete stimuli, and for each mutable guard discovered, a new test is recorded and added to the pool. Such iterative explorations begin a the reset state (application of initialization vectors) and are bounded by a predefined number of simulation cycles, defining the exploration length. Increasing the exploration length leads to increased concolic execution complexity as described in Section 2.3. The final optimal test is greedily derived (maximize branches covered per test) from the pool of individual tests such that all discovered branches are covered.

\subsection{Experimentation and Results}

This section evaluates the performance of our cycle-by-cycle concolic execution methodology against previous work [17, 25] that used hybrid concrete and symbolic execution. 


\subsubsection{Experiment Setup}

The test generation framework is written in Python (v3.4.3) and uses the Microsoft's Z3 (v4.5.1) [34] SMT solver. The design information parsed during preprocessing is stored and accessed as Python objects (Python pickling). The Verilated $\mathrm{C}++\mathrm{RTL}$ and the simulator harness which instantiates and provides accessor functions to the behavioral model of the design is compiled as shared library object that can be imported as a Python module. All experiments are run on a Linux (Lubuntu 15.10) machine with an Intel Core i7-975 (3.33GHz) with 6GB memory.

Table 3.2: Benchmark Description

\begin{tabular}{|c|c|c|c|c|c|}
\hline Benchmark & \#PI & \#PO & \#branches & \#gates & Unreach. \\
\hline \hline b01 & 4 & 2 & 26 & 45 & 0 \\
\hline b06 & 2 & 6 & 24 & 66 & 1 \\
\hline b10 & 11 & 6 & 32 & 172 & 1 \\
\hline b11 & 7 & 6 & 33 & 366 & 1 \\
\hline b14 & 32 & 54 & 211 & 3461 & 12 \\
\hline
\end{tabular}

HYBRO [17] and [25] are bounded test generators that begin at the reset state, and perform repeated concolic execution for a specific number of cycles. This limits the unrolling of the RTL and is a parameter to control the computation complexity of the symbolic evaluation effort. Since, we compare our work against these methods in this chapter, our test generation framework is built to perform bounded concolic execution. Our work is tested over the same benchmarks (Table 3.2) from ITC99 as used in [17]. Column 6 (Unreach.) in this table shows the number of branches that are found to be guaranteed unreachable during preprocessing. The cost of preprocessing which includes translation, parsing, static analysis and simulator compilation is at worst under $2 \mathrm{~s}$. 


\subsubsection{Branch Coverage}

Table 3.3 highlights the performance of our concolic execution methodology over HYBRO [17] and [25] in terms of branch coverage and runtime. Column 3-5 (BC\%) shows the overall branch coverage which is measured over all branch points. Column 6 (Reach. BC\%) shows our reachable branch coverage which discounts branches that are deemed guaranteed unreachable during preprocessing. Column 2 (Unroll Cycles) shows the degree of unrolling (same as [17]), and column 7 (Iter.) shows the number of iterations or rounds of concolic execution engaged. The number of rounds is decided experimentally per bound where it is initially set at 4 , and increased until a high branch coverage (>90\%) is consistently obtained. The column 8 (\#vec.) indicates the length of the final test that greedily covers all branches discovered in the test generation process. Information about test length was not provided in the compared works.

Table 3.3: Comparison of Branch Coverage

\begin{tabular}{|c|c||c|c|c||c|c|c|}
\hline \multirow{2}{*}{ Bench. } & \multirow{2}{*}{ Unroll Cycles } & \multicolumn{3}{|c||}{ BC $^{1 \%}$} & \multirow{2}{*}{ Reach. BC\% } & \multirow{2}{*}{ Iter. } & \multirow{2}{*}{$\#$ vec $^{2}$} \\
\cline { 2 - 5 } & & HYBRO & {$[25]$} & Ours & & & \\
\hline \hline b01 & 10 & 94.44 & 96.3 & 100 & 100 & 4 & 35 \\
\hline b06 & 10 & 94.12 & 96.3 & 95.83 & 100 & 4 & 39 \\
\hline b10 & 30 & 96.77 & 96.67 & 96.88 & 100 & 8 & 66 \\
\hline b11 & 10 & 78.26 & 81.82 & 93.94 & 96.875 & 16 & 68 \\
\hline b11 & 50 & 91.3 & 94.44 & 93.94 & 96.875 & 8 & 76 \\
\hline b14 & 15 & 83.5 & 98.95 & 92.89 & 98.49 & 36 & 506 \\
\hline
\end{tabular}

${ }^{1}$ Number of branches present in the RTL may vary between compared methods due to instrumentation differences.

${ }^{2}$ Test length not provided in [17] (HYBRO) and [25]

Minor differences in reachable coverage might be attributed to the different instrumentation methods used in compared works. Therefore, both overall coverage and reachable coverage from our work are reported. Our work generates tests that can reach more than $95 \%$ of all reachable branches across all compared benchmarks. Our methodology manages to cover all 
reachable branches in the case of b01,b06 and b10. In the design b14, we achieve $92.89 \%$ ( $98.5 \%$ reachable) branch coverage compared to HYBRO with $83.5 \%$ overall coverage. The test for $b 14$ is comparable to the search success of [25] (98.95\% reported branch coverage).

\subsubsection{Test Generation Runtime}

The runtime cost of our concolic execution based test generation effort is detailed in Table 3.4. The runtime is described in seconds in columns 3-6, and speedup of our work over previous methods in highlighted in column 6 and 7 .

Table 3.4: Comparison of Runtime

\begin{tabular}{|c|c||c|c|c||c|c|}
\hline \multirow{2}{*}{ Bench. } & \multicolumn{1}{|c|}{ Unroll Cycles } & \multicolumn{3}{c||}{ Time(s) } & \multicolumn{2}{c|}{ Speedup } \\
\cline { 3 - 7 } & & HYBRO & {$[25]$} & Ours & v/s HYBRO & v/s [25] \\
\hline \hline b01 & 10 & 0.07 & 0.55 & 0.01 & 7 & 55 \\
\hline b06 & 10 & 0.1 & 0.6 & 0.11 & 0.91 & 5.45 \\
\hline b10 & 30 & 52.14 & 24.61 & 0.39 & 133.69 & 63.10 \\
\hline b11 & 10 & 0.28 & 0.67 & 0.26 & 1.08 & 2.58 \\
\hline b11 & 50 & 326.85 & 270.28 & 1.21 & 270.12 & 223.37 \\
\hline b14 & 15 & 301.69 & 257.59 & 11.98 & 25.17 & 21.49 \\
\hline
\end{tabular}

The results in Table 3.4 show that our cycle-by-cycle concolic execution methodology is orders of magnitude faster than the compared work. The performance speedup may not be certifiable measurable at smaller designs like $b 01$ and b06, especially under relatively fewer cycles of unrolling. In the design b11, we see at low degree of unrolling (10 cycles), the performance is comparable. However, upon increasing the computation complexity, i.e. by raising the concolic execution effort to 50 cycles, it becomes clear that our lean methodology is superior to HYBRO $(270 \times$ speedup) and [25] (223× speedup). The remarkable improvement in execution costs of engaging a semi-formal technique like concolic execution reinvigorates the viability of constraint-based techniques in the RTL testing field currently dominated by search-based techniques. 


\section{Chapter 4}

\section{Test Generation using Factored Explorations}

Concolic execution on its own is hindered by the same limitations of path explosion and computational effort of evaluation over a large number of cycles. The entire Control Flow Graph (CFG) of the design is processed every cycle. As demonstrated in [32], factoring the exploration into a smaller number of cycles and combining the results of each exploration offers a promising avenue to scale. In this chapter, we present, CORT (Concolic RTL Test Generator), a methodology for RTL directed test generation that aims to maximize branch coverage with a minimal number of test vectors, in the shortest amount of time. Our work treats the test generation problem as a task of iteratively building the global Test Decision Tree (TDT) for the design over each exploration.

The following is a short overview of CORT's underlying test generation methodology. We iteratively build upon several relatively smaller explorations to generate the overall test. For each exploration, we perform a cycle-by-cycle concolic simulation to build the tests that reach branches that were not executed by the current concrete simulation, as described in 
Chapter 3. Firstly, the costly construction and solving of symbolic expressions is limited to primary input stimulated (activated) statements. Secondly, concrete values of unstimulated signals are substituted in expressions that use them. These measures reduce the cost of symbolic evaluation effort. In order to reveal alternate execution paths in the concrete simulation, the negated symbolic expressions for activated guard statements are solved using a formal Satisfiability Modulo Theory (SMT) solver, to generate mutation stimuli.

The concrete and mutation stimuli are used to build a test decision tree which represents the control-flow response of the design in the exploration. The decision trees from individual explorations incrementally uncover the overall control-flow response of the design to various stimuli over several cycles, i.e., a global Test Decision Tree. This is subsequently used to guide new explorations along heuristically determined starting system states. Once all explorations have concluded, the optimal test is derived directly from the global Test Decision Tree.

The rest of the chapter is organized as follows. Section 4.1 introduces the paradigm of the Test Decision Tree, along with its construction and interpretation. The CORT framework is described in detail and the example introduced in Chapter 3 is continued in Section 4.2. Finally, CORT is benchmarked and evaluated against previous work in Section 4.3.

\subsection{Test Decision Tree}

The test generation effort is represented as a decision tree in our work. Performing concolic execution over a given sequence of input vectors (concrete stimuli), we detect mutable guards and their corresponding mutation stimuli. The mutable guards can be considered as points of divergence in the control-flow, with the divergence being achieved by applying the mutation stimuli instead of concrete stimuli. 
Structurally, the decision tree consists of two types of nodes, data nodes and control nodes. A data node comprises zero or more concrete input vectors called default stimuli. Control nodes are non-terminal, and detail a cycle annotated mutable guard, its concrete execution (default execution) and mutation stimuli.

An exploration is the task of performing concolic execution over a given concrete stimuli and returning a decision tree representing its control-flow response. The exercise of building a decision tree begins with an empty data node. The concrete input vectors are enlisted cycle-by-cycle as default stimuli until their execution discovers a mutable guard. Thus, the data node leads to a new control node which will branch into two children, along edges called default and mutate, indicating the concrete and divergent execution of the mutable guard, respectively. The control node holds the mutation stimuli, and its branches represent a choice to either default or mutate (application of mutation stimuli) the control-flow. Subsequent concrete input vectors are enlisted into the default data nodes, branching as necessary until all concrete stimuli are exhausted.

Construction of the test represented by a data node in the decision tree requires a reverse traversal from that node towards the root gathering input vectors along the way. The default stimuli from each traversed data node are first collected in its entirety. Then, the mutation stimuli are merely noted from the control nodes that fell on the mutate branch of their parent during traversal. In situations where some mutation stimulus of a deeper control node coincides (same signal on the same cycle) with the mutation stimulus of a higher control node, the latter is ignored. Lastly, the default stimuli are overwritten by mutation stimuli for the cycles that it denotes. The task of overwriting the default stimuli with the mutation stimuli is indicative of the minimum change in the input vectors necessary to guide the control-flow to the target node. There is a need to apply the mutation stimuli over the complete default stimuli because the mutation of a guard (while honoring the control-flow 
through the guards above it) might require altering input, many cycles in the past.

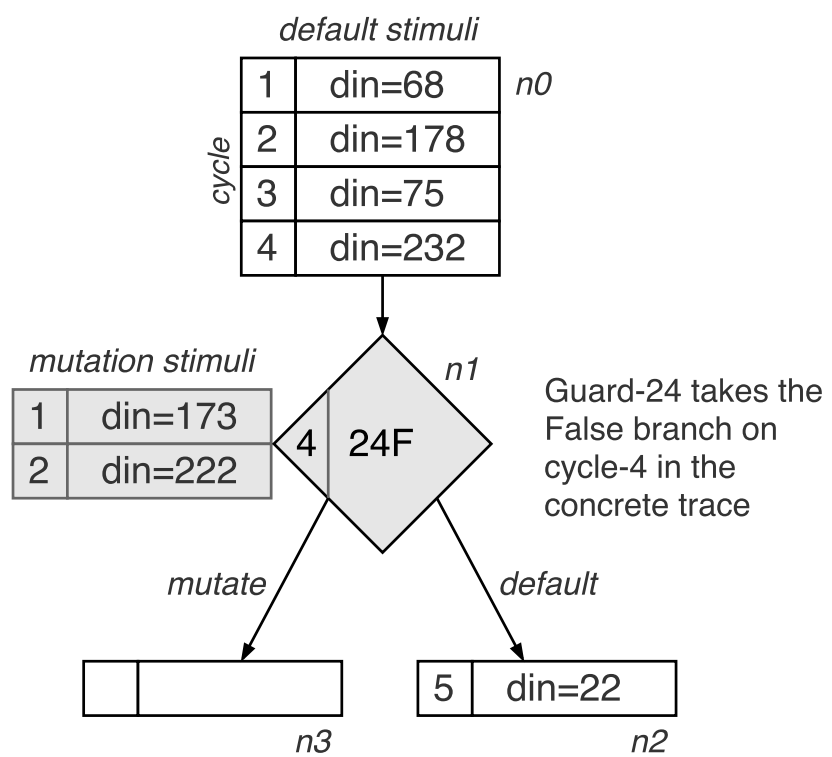

(a) TDT

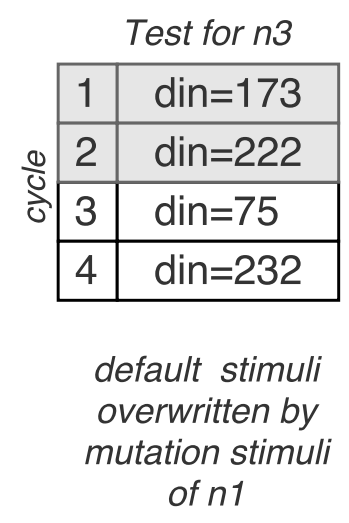

(b) Test to reach node $n 3$

Figure 4.1: Test Decision Tree for the example exploration in Section 3.3

Continuing the example in Section 3.3, the decision tree in Fig. 4.1a shows the response uncovered by the concolic execution based exploration. The test decision tree can be understood as an abstraction of the CFG unrolled over the simulated cycles, in terms of stimuli and response. In each cycle, a concrete input vector is applied to the system and a unique path is traversed in the CFG. However, the response to the stimuli may not be guaranteed in the same cycle, and in some cycles, there is no controllable response at all (cycle 1-3 in the example shown in Section 3.3). Thus we store the minimum amount information in terms of stimuli distributed over data and control nodes, describing the control flow response and means to alter it (mutation stimuli of control nodes). Data node $n 0$ in the TDT accumulates the first 4 concrete input vectors as no mutable guard was discovered before that point. In cycle 4, according to the concrete (default) execution the mutable guard-24 takes the False path. Control node $n 1$ records this control-flow response and the mutation stimuli that can alter it. The unexplored part of the control-flow response, n3 appears on the mutate edge 
of $n 1$. The remaining default stimuli is accumulated in data node $n 2$.

If the primary input $\operatorname{din}$ is modified as per the mutation stimuli of $n 1$ on cycles 1 and 2 in the decision tree, then the system would execute the True branch for guard-24 on cycle-4. This is well represented in the TDT by the test that reaches node $n 3$. From the TDT, we see that the test for $n 3$ is formed by $\langle n 0, n 1, n 3\rangle$. First, the default stimuli from the data nodes $n 0$ and $n 3$ are collected. Since, $n 3$ is currently empty, only $n 0$ can contribute its default stimuli. This action reveals the length of the test to be 4 cycle long. Next, the mutation stimuli from $n 1$ overrides the concrete stimuli on the appropriate cycles. Overall, a 4 cycle test (Fig. 4.1b) is constructed to reach node $n 3$.

\subsection{CORT Framework}

The framework for CORT is illustrated in Fig. 4.2. Identification of clocking and reset signals and initialization input vectors is expected from the user, along with the Verilog Hardware Description Language (HDL) source for the design. Currently, our work can handle multiple synchronous clocks, each of which can toggle at different rates. The user also configures a search strategy (consisting of several options) to be used for test generation.

Before test generation, a preprocessor generates a cycle-accurate functional simulator from the given Verilog alongside extracting various design information. The Test Generator (TG) block engages the Concolic Execution Engine (CEE) for several explorations towards the goal of building a test that achieves maximum branch coverage. The CEE receives input vectors from the TG and performs concolic execution over their concrete simulation. It discovers mutable guards and their mutation stimuli, and a returns a decision tree identifying them. The TG stitches these decision trees from individual explorations to form a global Test Decision Tree (TDT). At the start of each exploration, the TG selectively determines 


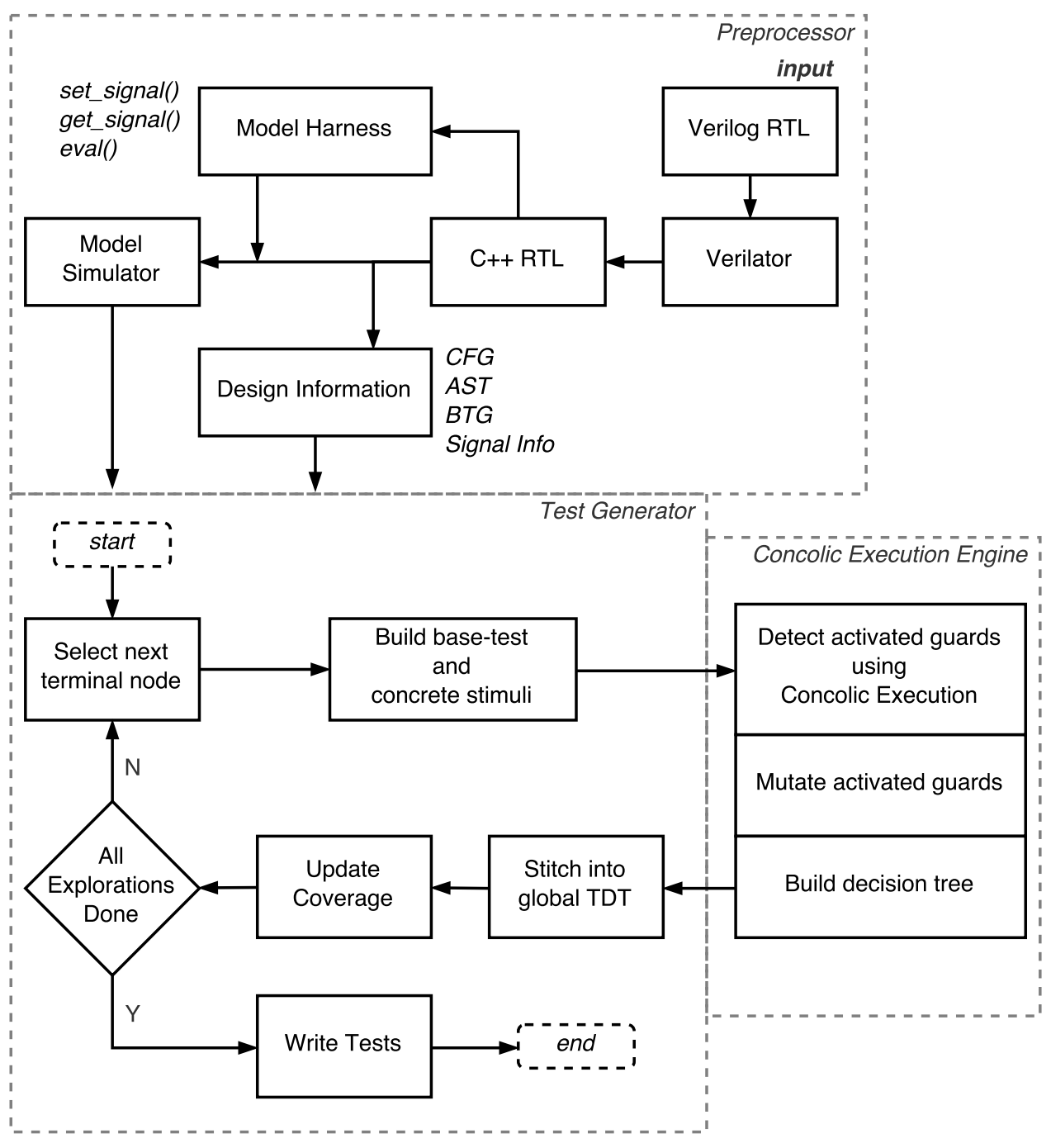

Figure 4.2: CORT Framework

terminal nodes in the TDT to commit new explorations from and generates appropriate input vectors to reach that node followed by random input vectors for the unexplored cycles. Once all explorations have completed, the input vectors that form the optimal test are written out. The working of the CORT framework is described over the example introduced in Section 3.2.2. 


\subsubsection{Preprocessing}

The Preprocessor performs the translation and analysis tasks described in Section. 3.2. The Verilog RTL source is translated to $\mathrm{C}++$ and harnessed to produce a cycle-accurate functional simulator. The Abstract Syntax Tree (AST) and Control Flow Graph (CFG) [9] is extracted during this phase along with specifications for various signals in the design. To avoid redundant illustration, the translated $\mathrm{C}++\mathrm{RTL}$ for the example design introduced in Section 3.2.2 and its behavioral CFG is shown in Fig. 3.2b and Fig. 3.3a respectively.

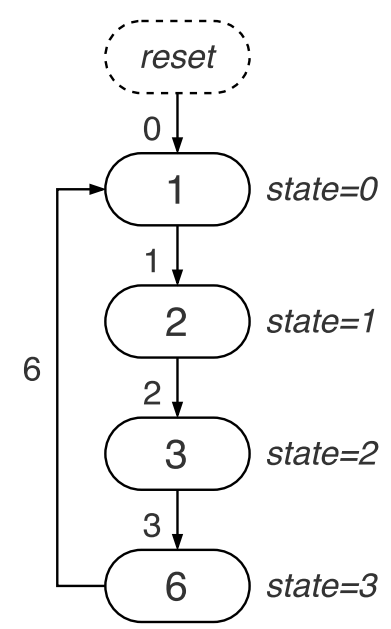

Figure 4.3: Branch Transition Graph

RTL for FSM-based designs often involves guidepost variables to denote the state of the system. In CORT, we extend the preprocessing task towards the static analysis of assignments and guards for such variables in the AST to construct a Branch Transition Graph (BTG) as described in [31]. A node in the BTG is representative of a state in an FSM graph, and its branch coverage point identifies the guard that acts as its state-variable guidepost. The BTG edges denote the branch that must be covered to transition from the current state to the next. We can associate all branch points with their respective BTG nodes, by following their hierarchy in the CFG. This task mimics the purpose of design abstraction in [32] and aims to identify the underlying design FSM. The BTG representation of the FSM in the 
example design is shown in Fig. 4.3.

\subsubsection{Concolic Execution Engine}

The Concolic Execution Engine (CEE) is the heart of the CORT framework and performs the primary task of exploring the design response for a given sequence of input vectors. It follows the same core concolic execution methodology as described in Section 3.3. The Test Generator (TG) provides the CEE with a sequence of concrete input vectors (column 2 in Fig. 3.1) to be used for a new exploration along with a base test sequence which brings the simulated design to a desired state.

Prior to simulation during both phases of our cycle-by-cycle concolic execution, initialization vectors and the base test are applied to the model. Concolic execution is only performed on the exploration vectors, and not on the initialization and base test vectors. The CEE simulates the concrete input and symbolically evaluates the trace to discover mutable guards, and their mutation stimuli. In our example, that would be guard-24 on cycle- 4 with a mutation stimuli of $\operatorname{din}_{1}=173$ and $\operatorname{din}_{2}=222$ which allows the test to progress to the True branch (branch 4). Once the concolic analysis is completed, the CEE constructs a decision tree (Fig. 4.1a) for the current exploration as per Section 4.1 and returns it to the Test Generator.

\subsubsection{Test Generation}

The Test Generator (TG) block is primary controller for the entire test generation strategy which fundamentally revolves around incrementally discovering and growing the control flow response of the design-under-test in the form of a Test Decision Tree (TDT). It seeds the Concolic Execution Engine (CEE) with concrete stimuli and analyzes its response to grow 
the global TDT. The TG is also responsible for the selection of system states to start each new exploration from.

A configurable parameter called exploration-radius $(R)$ determines the length of each exploration. An iteration begins by heuristically selecting a terminal data node in the TDT. Assume that the terminal data node $n 3$ from Fig. 4.1a is selected as the starting point for a new exploration. Reaching the system state represented by $n 3$ requires that the mutate branch is taken from node $n 1$. The test is constructed as described in Section 4.1 where the entire 4-cycle default stimulus is collected first, and then overwritten by specific mutation stimuli on cycle 1 and 2 . The execution region under the then branch of guard-24 on cycle- 4 is unexplored, which is also implied by an empty n3. Thus, to ensure that the exploration does not miss any mutable guard on cycle-4 along the new execution branch, the last input vector in the 4-cycle test that reaches $n 3$, is set as the first cycle of the concrete stimuli. This represents a 1-cycle overlap of the previous exploration and the new one.

The concept of stitching factored decision trees that guide the overall control flow of the design is afforded by overlapping explorations using a configurable parameter, explorationoverlap $(Q)$. The exploration-overlap can take a minimum value of 1 . The overlapping vectors are treated as concrete stimuli, which implies that concolic execution is performed over $Q+R$ vectors. The test that reaches the selected terminal node is reduced by $Q$ cycles to form a base-test, and the remaining vectors are used as overlap. For the new exploration's concrete stimuli, the $Q$ overlapping vectors are supplemented by $R$ randomly generated input vectors (with inactive reset input).

For our example, assume an $R=10$. The cumulative 3 -cycle base-test and the new 11cycle $(Q+R)$ concrete stimuli with $Q=1$, driving the new exploration at $n 3$, is shown in Fig. 4.4a. The returned decision tree in displayed in Fig. 4.4b. This time, the previously empty $n 3$ starts off as the head node while building the exploration's decision tree. However, 


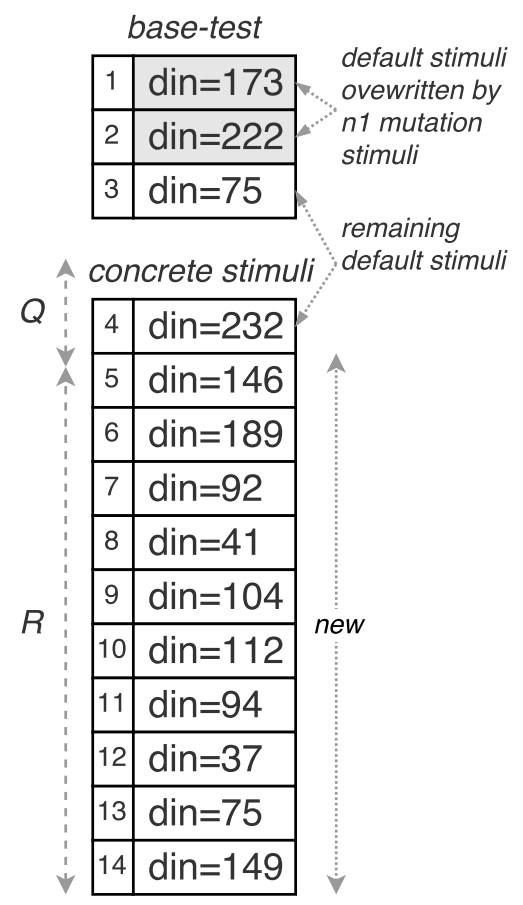

(a) Concrete Stimuli

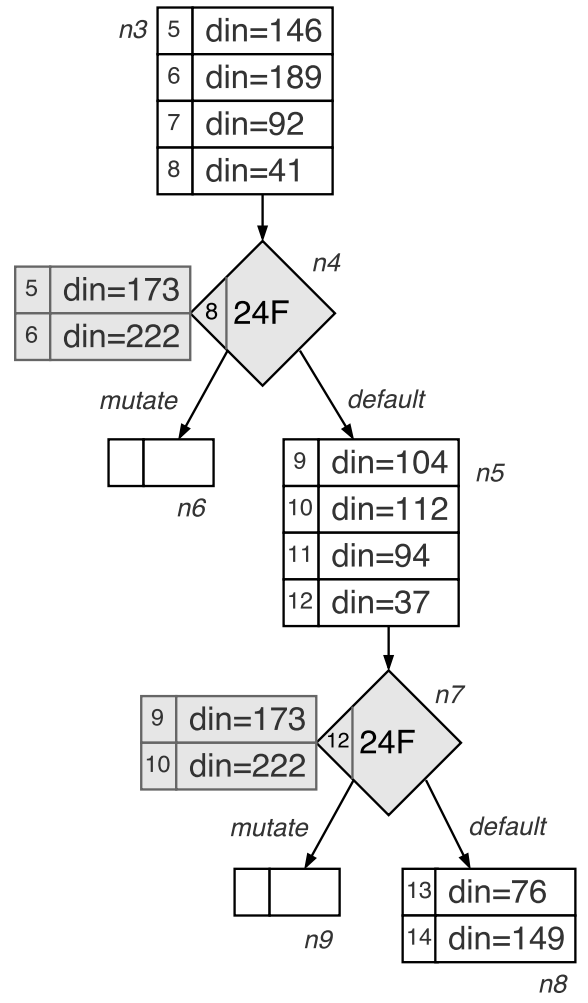

(b) Control-Flow Response

Figure 4.4: New exploration at $n 3$

the vectors that were included as overlap are not gathered as default stimuli, as illustrated by the absence of $\langle$ cycle-4, din=232 $>$ in the updated $n 3$. The stitching of the decision tree returned by the new exploration at $n 3$ is shown in Fig. 4.5.

Overlapping explorations may lead to re-discovering mutable guards within the region of overlap. The CEE ignores these previously explored cycle-specific mutable guards while building its local decision tree, but includes them for mutation effort (in cases where their stimuli intersect with activated guards down the new concrete path). Increasing $Q$ may increase the discovery of mutable guards in the new exploration, as it deepens the influence of primary input stimuli. A $Q=\infty$ would rediscover all mutable guards from the root of the TDT but would be computationally intensive. A smaller $Q$ and $R$ would lead to relatively fewer activated statements being processed per exploration. 


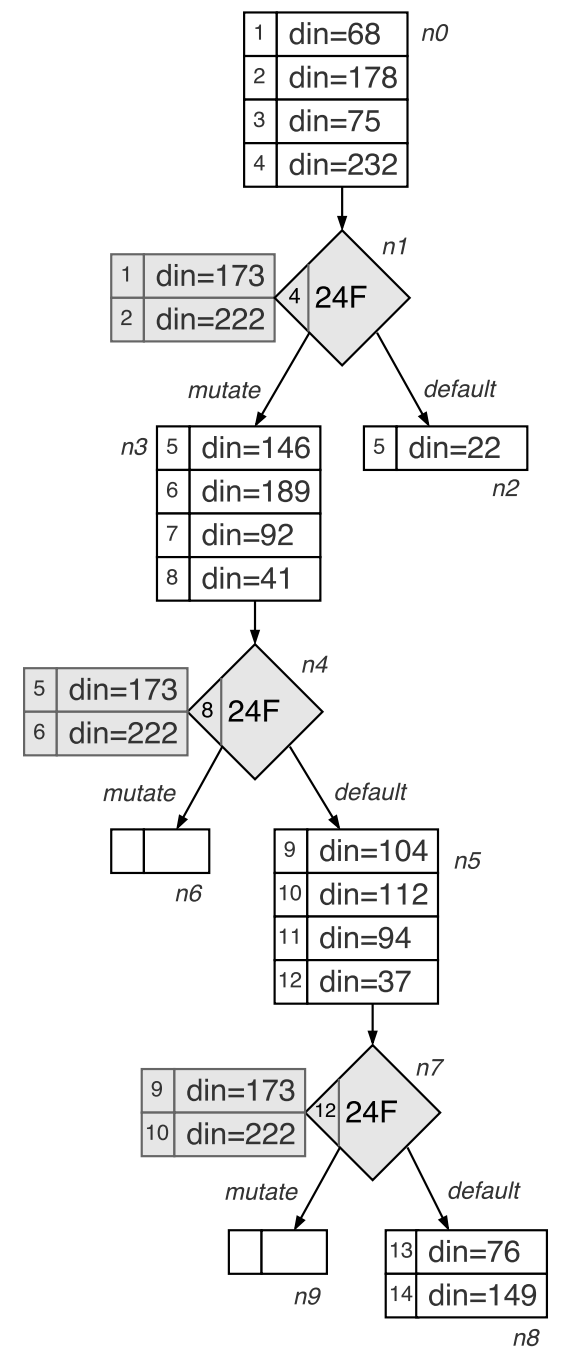

Figure 4.5: Global Test Decision Tree after two explorations

\subsubsection{Systematic Exploration}

The test represented by a data node includes the test that reaches it, and its default stimuli (if any). The test representing node $n 6$ is shown in Fig. 4.6a. At the end of an exploration, the coverage status for each new terminal node is recorded by simulating the design over their tests. If no mutable guards were discovered during an exploration, then the head data node from which the exploration began would continue to be terminal, though with more default stimuli. The coverage status needs to be refreshed for such updated terminal nodes. 


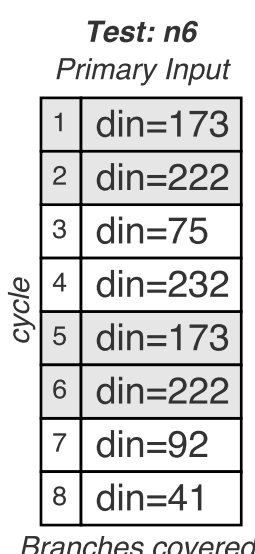

Branches covered: $\{0,1,2,3,4,6,7\}$

(a) Test for node $n 6$
Final Test

Primary Input
\begin{tabular}{|l|l|}
\hline 0 & reset $=1$ \\
\hline 1 & din $=173$ \\
\hline 2 & din $=222$ \\
\hline 3 & din $=75$ \\
\hline 4 & din $=232$ \\
\hline 5 & din $=146$ \\
\hline 6 & din $=189$ \\
\hline 7 & din $=92$ \\
\hline 8 & din $=41$ \\
\hline
\end{tabular}
Branches covered:

$\{0,1,2,3,4,5,6,7\}$

(b) Final test output

Figure 4.6: Tests derived from the TDT and their branch coverage

The TG currently employs three heuristic metrics to select the next terminal node for each exploration.

- Random Path Selection (RPS): This selection heuristic was introduced in KLEE [5]. A terminal node is selected by traversing the TDT, starting from the root with random choices made at every branch. This strategy is expected to uncover easy-to-reach system states. Despite being shallow heuristic, a random selection based on TDT traversal is immune to the effects of loops and state space traps.

- Coverage Oriented Selection (COS): This strategy aims to guide the exploration along least covered branches in hopes of uncovering unexplored regions of the design. Say, $I$ branches are covered among all $J$ terminal nodes. And, $c_{i j}$ is the coverage count for branch $i$ in terminal node $j$. Then, as shown below, a score $s_{j}$ for each node is calculated from a branch heuristic weight $w_{i}$. The node with the highest score is 
selected.

$$
\begin{aligned}
C_{i} & =\sum^{\forall j \in J} c_{i j} \\
w_{i} & =\frac{\sum^{\forall i \in I} C_{i}}{C_{i}} \\
s_{j} & =\sum^{\forall i \in I \mid c_{i j}>0} w_{i}
\end{aligned}
$$

- Target Oriented Selection (TOS): Given a target branch, TOS restricts a COS type heuristic selection to terminal nodes that may possibly reach the target. If a terminal node is found to cover a branch that offers no path to the target branch in the design BTG, then it is ignored during selection. Such ignored nodes denote tests that proceed the control flow along paths of the FSM away from the target state. Restricting the coverage oriented heuristic to viable nodes has a better chance of guiding the test generation effort towards the target.

The concluding task for the Test Generator (TG) is to derive the test from the global Test Decision Tree (TDT). Writing out the optimal test can be boiled down to selecting the optimal set of data nodes in the TDT that cover all explored branches with the minimal number of input vectors. The technique of deriving the optimal test from the TDT removes the requirement to use standard test compaction techniques [23], thereby further reducing the overall functional testing time.

We utilize a greedy approach for handling this operation. With a breadth-first traversal, we collect a list of data nodes, $M$, that were the first at discovering a branch, until $M$ includes nodes that cover all discovered branches, $I$. The test, $T_{j}$ represented by each node- $j$ includes the test that reaches it and its default stimuli. Algorithm 1 shows the greedy accumulation of vectors among $M$, to build the final test, $T$. Concluding our example, the final test written 
out will only consist of $n 3$ as it covers all branches (specifically, branch- 4 in cycle- 4 , and branch-5 in cycle-8). A test of length, 9 (Fig. 4.6b) is built concatenating the initialization vector and the 8-cycle test represented by $n 3$.

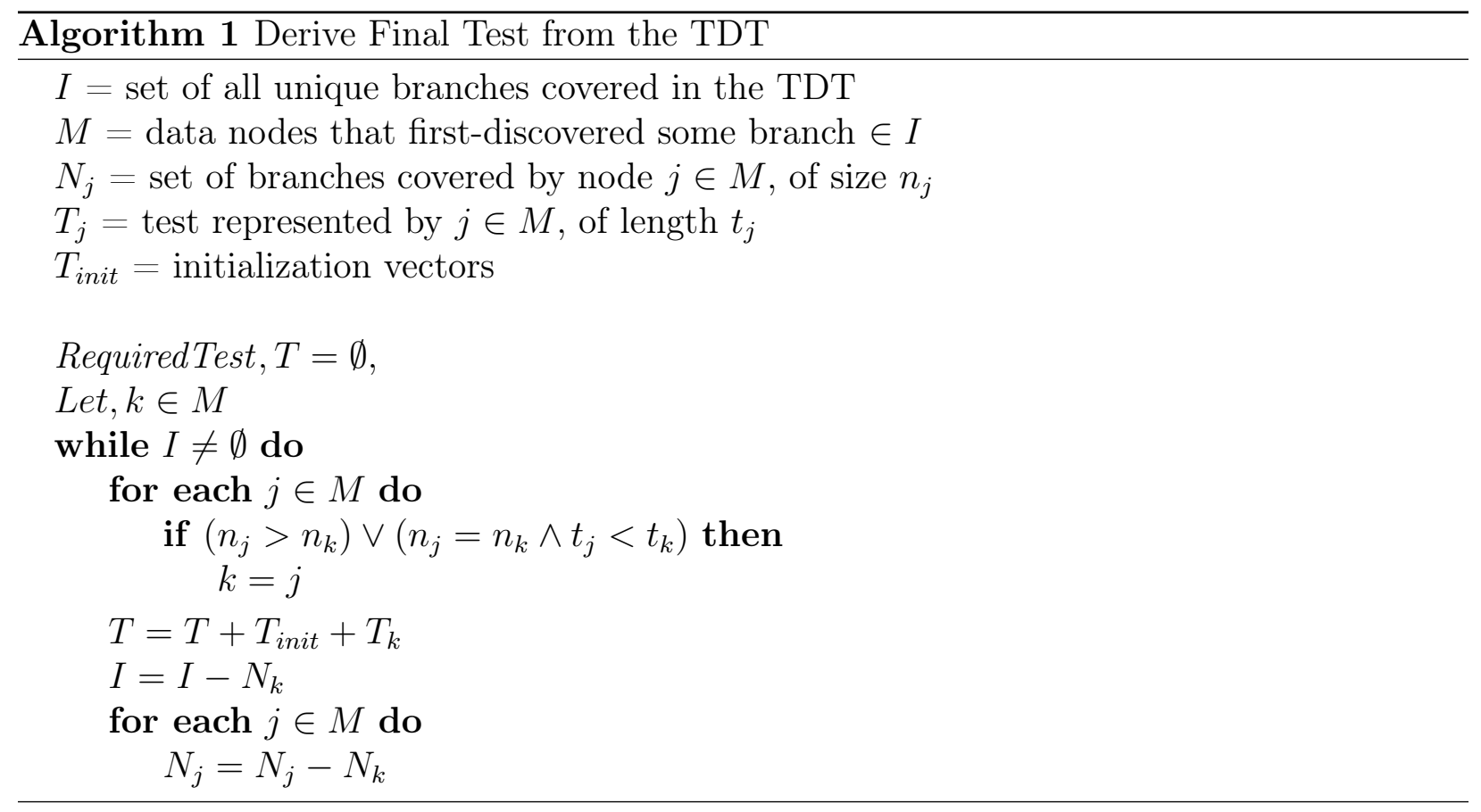

\subsection{Experimentation and Results}

CORT is written in Python (v3.4.3) and uses the Z3Py Python interface to Z3 (v4.5.1) [34], an SMT solver from Microsoft Research. The design information parsed and generated by the Preprocessor is stored as Python objects (Python pickling) in binary format to be read in further stages. The simulator source code which includes the Verilator $\mathrm{C}++$ translation of the RTL and its harness generated by the Preprocessor is compiled as shared library object that can be imported as a Python module. All experiments are run on a Linux (Lubuntu 15.10) machine with an Intel Core i7-975 (3.33GHz) with 6GB memory. We present CORT's performance in terms of branch coverage, test length and execution time over benchmarks 
from ITC99 [8] (row 2-8) and IWLS-2005 [4] (row 9-14) detailed in Table 4.1.

Table 4.1: Benchmark and CORT Strategy

\begin{tabular}{|c|c|c|c|c|c|c|c|c|c|}
\hline Bench. & PI & PO & Gates & Bran. & Unreach. $^{1}$ & Pre.(s) & Q & R & Search \\
\hline \hline b06 & 2 & 6 & 66 & 24 & 1 & 1.9 & 1 & 8 & RPS \\
\hline b07 & 1 & 8 & 382 & 19 & 1 & 1.98 & 1 & 8 & RPS $+16 x C O S$ \\
\hline b10 & 11 & 6 & 172 & 32 & 1 & 1.98 & 4 & 8 & RPS $+32 x C O S$ \\
\hline b11 & 7 & 6 & 366 & 33 & 1 & 1.92 & 4 & 8 & RPS $+128 x C O S$ \\
\hline b12 & 5 & 6 & 1000 & 105 & 1 & 2.12 & 1 & 128 & $\begin{array}{c}\text { RPS }+ \text { TOS }(c 1) \\
+ \text { TOS }(c 2)\end{array}$ \\
\hline b14 & 32 & 54 & 3461 & 211 & 12 & 2.14 & 8 & 8 & RPS $+128 x C O S$ \\
\hline b15 & 36 & 70 & 6931 & 149 & 1 & 2.15 & 8 & 16 & RPS $+128 x C O S$ \\
\hline ss_pcm & 17 & 9 & 470 & 19 & 0 & 1.99 & 2 & 4 & RPS $+128 x C O S$ \\
\hline usb_phy & 13 & 18 & 546 & 144 & 0 & 2.1 & 4 & 8 & RPS $+192 x C O S$ \\
\hline sasc & 14 & 12 & 549 & 58 & 0 & 1.99 & 4 & 8 & RPS $+128 x C O S$ \\
\hline simple_spi & 14 & 12 & 821 & 81 & 1 & 1.98 & 4 & 8 & RPS $+128 x \operatorname{COS}$ \\
\hline i2c & 16 & 14 & 1142 & 112 & 0 & 2.07 & 4 & 20 & RPS $+192 x C O S$ \\
\hline spi & 45 & 45 & 3227 & 89 & 0 & 2.02 & 4 & 8 & RPS $+192 x C O S$ \\
\hline
\end{tabular}

${ }^{1}$ Statically determined unreachable branches (Section 3.2)

\subsubsection{Search Strategy}

The benchmark circuits used and the parameters set for each circuit are described in Table 4.1. The number of branches instrumented by Verilator is shown in column 4 (Bran.). During preprocessing, we also statically analyze the use-definition chains in the parsed Abstract Syntax Tree to determine unreachable branches (column 6) as described in Section 3.2. These may not include all possible unreachable branches. The one-time cost for preprocessing inclusive of the simulator compilation is shown in column 7 (Pre.). The individual exploration for each design is bound by exploration-radius $(R)$ and exploration-overlap $(Q)$, selected as per design complexity and size. These parameters are experimentally determined with a minimum of $Q=1$ and $R=4$ and are increased until the trials for test generation results in high branch coverage (aim: $>95 \%$ ). 
Search in column 10, describes the quantity and type of the iterative selection method for each exploration. We begin growing the TDT for each design with repeated rounds of RPS until no new coverage points are discovered (for four rounds). The actual number of RPS iterations during test-generation is non-deterministic and thus is simply mentioned as RPS. This is usually followed by multiple explorations using COS, selected as per design size. A description of $R P S+N \times C O S$ implies that $\mathrm{N}$ explorations using the COS strategy were pursued after exhausting RPS. Almost all designs are suitably covered by using RPS and COS alone. For b12, a single player game of guessing a sequence, a TOS for the branches representing hard-to-reach states c1 and c2 [20,32] is repeated, until the targets are covered. A relatively larger exploration radius is set for b12 because the primary inputs are only used in guards, offering low concolic execution complexity.

\subsubsection{Branch Coverage}

CORT results are compared against HYBRO [17], [25] and BEACON [20] in Table 4.2. For unreported benchmarks in HYBRO and [25], $N A$ is placed. Columns 5 and 11 highlights the branch coverage (overall) and test length for CORT. Column 9 describes the time taken by CORT for executing the search strategy and generating the test for each benchmark and excludes the preprocessing cost. Column 12 (Reach. BC\%) represents the CORT's branch coverage only accounting for reachable branches. A quick glance shows that our work, despite relying on interleaved concrete and symbolic constraint solving, demonstrates significantly

shorter test generation time and achieves a high RTL branch coverage. A fewer number of vectors are generated for the same goal of maximal branch coverage compared to BEACON, a search-based test generator. The minor differences in coverage between tools could be attributed to instrumentation differences and optimizations. 
Table 4.2: Performance Comparison with Previous Work

\begin{tabular}{|c|c|c|c|c|c|c|c|c|c|c|c|}
\hline \multirow{2}{*}{ Bench. } & \multicolumn{4}{|c|}{ Branch Coverage $^{1} \%$} & \multicolumn{4}{|c|}{ Run Time(s) } & \multicolumn{2}{|c|}{ \#Vectors $^{2}$} & \multirow{2}{*}{$\begin{array}{l}\text { Reach. } \\
\text { BC\% }\end{array}$} \\
\hline & HYBRO & [25] & BEACON & CORT & HYBRO & [25] & BEACON & CORT & BEACON & CORT & \\
\hline b06 & 94.12 & 96.3 & 95.83 & 95.83 & 0.1 & 0.55 & 0.0054 & 0.12 & 1731 & 18 & 100 \\
\hline $\mathrm{b} 0$ & & $\mathrm{~N}$ & & 90 & $\mathrm{~A}$ & NA & & & 759 & 46 & 94.74 \\
\hline & & 96.77 & & 6.88 & 214 & 24.61 & & & 3547 & 28 & 100 \\
\hline b11 & & 94.44 & & 9 & 326.85 & 270.28 & & 3.11 & 1213 & 430 & 100 \\
\hline $\mathrm{b} 12$ & NA & NA & 98.09 & 98.09 & NA & NA & 111.42 & 35.23 & 37006 & 35081 & 99.04 \\
\hline b14 & 83.5 & 98.95 & 91.94 & 93.36 & 301.69 & 257.59 & 204.65 & 40.98 & 4381 & 573 & 98.99 \\
\hline
\end{tabular}

${ }^{1}$ Number of branches present in the RTL may vary between compared methods due to instrumentation differences. Represents overall branch coverage.

2 Test length was not reported for HYBRO [17] and [25].

${ }^{3}$ Reachable branch coverage for CORT

Consider design $b 10$ where CORT achieves a high branch coverage score (96.88\% overall, $100 \%$ reachable branches) on par with HYBRO and [25] in only 0.52s. Although HYBRO, [25], and CORT base their test generation effort on hybrid concrete and symbolic simulation, our work is more than $40 \times$ faster for $b 10$ alone. For b14, we compare CORT with BEACON which also uses Verilator for instrumentation and simulation. We achieve a higher coverage ( $93.36 \%$ overall, $99 \%$ reachable branches) with a test which is $7.5 \times$ smaller.

Concerning the target-oriented test generation, CORT consistently manages to cover states $\mathrm{c} 1$ and $\mathrm{c} 2$ in 612 . Satisfying $\mathrm{c} 1$ requires timing-out by not pressing the input $(k)$. The deepest system state in b12, c2 (WIN, state variable gamma $=25)$, which requires over 30000 cycles in a comparable time to PACOST [32] (single-cycle: 54.22s, multi-cycle: 169.83s). Reaching the winning state requires guessing the correct input over 500 times in sequence. Pressing the wrong key in the sequence traps the system in LOSS (state variable gamma $=23$ ) unless reset or restarted. Thus, nodes in the TDT that have reached states that offer no path to $\mathrm{c} 2$ in the BTG for 612 are ignored during selection for new TOS(c2) explorations. Nonetheless, the TDT represents the entire control-flow response of the system, and thus the final test is written out covers $\mathrm{c} 1, \mathrm{c} 2$, and LOSS. Concolic execution based directed test generators that only rely on fixed unrolling like HYBRO and [25] are unable to reach such deep targets. 
Table 4.3: Extended Performance Comparison

\begin{tabular}{|c|c|c|c|c|c|c|c|c|}
\hline \multirow{3}{*}{ Bench. } & \multicolumn{4}{|c|}{ Stochastic Search-Based } & \multirow{2}{*}{\multicolumn{3}{|c|}{$\begin{array}{c}\text { Hybrid Concrete } \times \text { Symbolic } \\
\text { CORT }^{2}\end{array}$}} & \multirow{3}{*}{$\begin{array}{c}\text { Reach. }^{3} \\
\text { BORT } \\
\text { BC\% }\end{array}$} \\
\hline & \multirow{2}{*}{$\begin{array}{c}\mathrm{RAND}^{1} \\
\mathrm{BC} \%\end{array}$} & \multicolumn{3}{|c|}{ BEACON } & & & & \\
\hline & & $\mathrm{BC} \%$ & Vec. & Time(s) & $\mathrm{BC} \%$ & Vec. & Time(s) & \\
\hline $\mathrm{b} 15$ & 42.13 & 89.93 & 38234 & 6.88 & 92.62 & 367 & 27.7 & 93.24 \\
\hline ss_pcm & 100 & 100 & 393 & 2.98 & 100 & 61 & 2.76 & 100 \\
\hline usb_phy & 82.64 & 82.64 & 3918 & 5.67 & 84.72 & 764 & 75.15 & 84.72 \\
\hline sasc & 91.67 & 91.67 & 278 & 4.06 & 91.67 & 68 & 16.48 & 91.67 \\
\hline simple_spi & 98.3 & 97.53 & 5566 & 4.32 & 98.77 & 663 & 104.2 & 100 \\
\hline $\mathrm{i} 2 \mathrm{c}$ & 83.27 & 87.5 & 6445 & 5.01 & 87.5 & 1188 & 430.38 & 87.5 \\
\hline spi & 90.31 & 100 & 1180 & 7.36 & 100 & 71 & 28.6 & 100 \\
\hline
\end{tabular}

1 Test consists of random input vectors generated for $50 \mathrm{k}$ cycles

${ }^{2}$ CORT, written in Python is compared against $\mathrm{C}++$ based BEACON

${ }^{3}$ Reachable branch coverage for CORT

Table 4.3 shows results of test generation on the remaining benchmarks for which results for HYBRO and [25] were not available. Hence, we compare CORT against random test generation (RAND), and BEACON configured to the settings used in [20]. RAND is constrained to non-reset primary inputs for 50000 cycles and averaged across eight trials to represent a baseline. The table highlights the best results from BEACON and CORT. BC\% (overall), Vec. and Time represent percentage branch coverage, test length and runtime respectively. Reach. BC\% describes CORT's branch coverage without accounting for guaranteed unreachable branches.In all cases, the result from CORT matches or exceeds the compared methods in terms of coverage and test length. The design, b15 contains a 16-byte prefetch queue (array) through which both instructions (80386 opcodes) and data are buffered. Random test generation is unable to handle such sequential depth in the control-flow. BEACON almost covers as many branches (89.93\%) as CORT (92.62\% overall, $93.24 \%$ reachable) but requires notably more vectors to do so (almost 100×).

Although CORT is not as fast as stochastic search-based techniques like BEACON for these circuits, the tests generated are undoubtedly efficient in terms of coverage per vector. This is within our expectation, as CORT is a Python-based semi-formal methodology relying on 
costly SMT operations. Nonetheless, in comparison to the same class of techniques that use hybrid concrete and symbolic simulation (HYBRO, [25]), CORT is remarkably faster. These results are indicative of CORT's ability to generate minimal directed tests for practical Verilog designs.

\subsubsection{Effects of Exploration Length}

Table 4.4: Effects of Exploration Length for b15

\begin{tabular}{|c|c|c|c|c|c|}
\hline $\mathrm{Q}$ & $\mathrm{R}$ & $\mathrm{Q}+\mathrm{R}$ & $\mathrm{BC} \%$ & Vec. & Time(s) \\
\hline \hline 2 & 2 & 4 & 84.73 & 288 & 2.13 \\
\hline 4 & 4 & 8 & 88.42 & 261 & 4.62 \\
\hline 8 & 8 & 16 & 90.60 & 374 & 9.82 \\
\hline 8 & 16 & 24 & 91.44 & 426 & 16.57 \\
\hline 16 & 16 & 32 & 91.52 & 401 & 20.51 \\
\hline 16 & 32 & 48 & 91.61 & 472 & 40.73 \\
\hline
\end{tabular}

In Table 4.4 , for a fixed search strategy of $64 \times C O S$, the exploration radius $(R)$ and exploration overlap $(Q)$ are varied for the design, b15. An increasing exploration length $(R+Q)$ is analyzed, and the average branch coverage $(B C \%$, overall), test length (Vec.) and runtime (Time) are reported over 8 trials. Our experiments show that a suitably sized exploration is necessary for generating efficient tests. With shorter exploration lengths (row 2-3), we discover fewer branches in the same search. However, these tests require fewer vectors to cover those branches, highlighting the utility of deriving the overall test from the TDT. Finding the suitable exploration length is a matter of experimentation and knowledge of design complexity. A smaller $Q$ leads to discovering fewer mutable guards per exploration and thus, longer tests, which is indicative of a greater reliance on the randomness of the concrete stimuli $(R)$ to reach certain branches. On the other hand, an exploration with a high $Q$ is computationally intensive and demands a high execution time. 
It is seen that an exploration length of 24 (row 5) optimally leverages all goal metrics. The efficiency of the test generation effort stagnates beyond a certain an exploration length, indicating its sufficiency at handling the design complexity. Lastly, row $7(Q=16, R=32)$ shows a larger final test length, which is an adverse effect of the greedy test accumulation technique. 


\section{Chapter 5}

\section{Conclusion}

\subsection{Limitations and Future Work}

In our work, the concrete values of variables can only be read between, not during, cycles. In the same cycle, if a variable is used in a statement between two blocking definitions of the same variable, then no valid (pre/post cycle) concrete values can read for its use. When the CEE encounters such a situation in the concrete path, it is reported to the user, and the program terminates. The user is expected to insert a temporary internal variable in the RTL to mirror the first blocking definition. This adds no functional value and does not increase the size of the synthesized design, but is quite helpful for concolic execution. Converting the C ++ RTL to Static Single Assignment (SSA) form would solve this problem, and is expected in our future work.

Secondly, our work is limited to RTL designs containing signals with a maximum width of 64 bits. This limitation is posed by Verilator's handling of large-width signals as contiguous C arrays. The capability to handle such signals and their assignments is also being considered 
for future work. In hardware RTL, the sequential memory elements like registers usually change their values are their trigger clock edges. In our methodology, the RTL is translated to $\mathrm{C}++$, where this RTL feature (non-blocking statements in Verilog) is mimicked by using temporary variables which are function scoped (and not declared at the top class level). This requires extended evaluation effort where the RTL Control Flow Graph is analyzed to infer resolution of these temporary variables and see their effect in actual named signals (available at the top class). The immediate solution to this would be to scope these temporary variables to the top simulator class, but would required severe modification to the Verilator compiler source code.

Lastly, CORT is written in Python as a proof of concept and will be reconstructed in $\mathrm{C}++$ for performance.

While developing our work we curated certain ideas that could help enhance concolic execution based testing methodology. They are listed as follows.

- While the Test Decision Tree (TDT) excellently abstracts the control-flow response of the design in terms of stimuli, it does not account for system states. In reality, designs have a limited number of functionally reachable system states, and these are often revisited. The TDT needs to be extended to support the identification of system states, possibly abstracted to control signals. Additional edges should be added showing a loop of the control flow back to previously visited system states, implying the response needs to be recorded in the form of a graph rather than a tree.

- Upon simulation, the control flow executed through a unique path in the CFG. A cost metric that is based on maximizing all unique single-cycle paths discovered could be a better search heuristic than branch coverage alone. Single-cycle path coverage encompasses branch coverage and may offer more differentiation between solutions 
that need to be selected.

\subsection{Conclusion}

In this thesis, we proposed a novel concolic execution methodology for the Register Transfer Level (RTL). We strongly intertwine symbolic execution and concrete simulation, and dynamically construct symbolic constraints in a cycle-by-cycle functional simulation. Our symbolic analysis is lean and restricted to primary input stimulated regions of the RTL. Traditionally, a path constraint would be built over the entire concrete trace, extracted after concrete simulation. However, the simulator used in our work is instrumented for direct access of all signals in the design at any time. Furthermore, the simulator is enhanced to record the concrete trace in terms of branch IDs during simulation. Unstimulated variables in statements are concretely read from the simulator and substituted in-place to construct symbolic expressions. This reduces the overall computational complexity of our formal analysis component. The base cycle-by-cycle concolic execution methodology is tested against previous work and is demonstrated to be significantly superior.

We have presented CORT; a factored concolic execution based test generation technique for the Register Transfer Level. Our method tightly interleaves concrete simulation and symbolic evaluation at a cycle-by-cycle level, providing advantageous performance over previous hybrid techniques. Our minimalist approach to concolic execution, where we dynamically evaluate primary-input stimulated statements, allows us to explore the search space effortlessly. Furthermore, we abstract the control-flow response for the entire test search effort in a novel representation, the Test Decision Tree (TDT), which is systematically grown over several factored explorations. We not only use the TDT for strategic guidance for each new exploration but also derive the optimal test from it without requiring any standard test com- 
paction techniques. Our results show that CORT generates smaller tests with high branch coverage, faster than existing hybrid semi-formal methods. 


\section{Bibliography}

[1] A. Koelbl, J. H. Kukula, and R. Damiano, "Symbolic RTL simulation," in Proc. of Design Automation and Test Europe Conference, 2001, pp. 47-50.

[2] A. Biere, A. Cimatti, E. M. Clarke, and Y. Zhu, "Symbolic model checking without BDDs," in Proc. of International Conference on Tools and Algorithms for Construction and Analysis of Systems, 1999, pp. 193-207.

[3] B. Beizer, "Software testing techniques (2nd ed.)," Van Nostrand Reinhold Co., USA, 1990.

[4] C. Albrecht, "IWLS 2005 Benchmarks," International Workshop on Logic Synthesis, 2005.

[5] D. Dunbar, C. Cadar, and D. Engler, "KLEE: unassisted and automatic generation of high-coverage tests for complex systems programs," in Proc. of the 8th USENIX conference on Operating Systems Design and Implementation, 2008, pp. 209-224.

[6] D. Krishnaswamy, M. S. Hsiao, V. Saxena, E. M. Rudnick, J. H. Patel and P. Banerjee, "Parallel genetic algorithms for simulation-based sequential circuit test generation," in Proc. of Tenth International Conference on VLSI Design, Hyderabad, 1997, pp. 475-481. 
[7] E. Clarke, A. Biere, R. Raimi, and Y. Zhu, "Bounded model checking using satisfiability solving," Formal Methods in System Design, 2001, pp. 7-34.

[8] F. Corno, M.S. Reorda, G. Squillero, "RT-level ITC'99 benchmarks and first ATPG results," in Proc. of the IEEE Design and Test of Computers, 2000, vol. 17, no. 3, pp. 44-53.

[9] F.E. Allen, "Control flow analysis," SIGPLAN Not., 1970, vol. 5, no. 7, pp. 1-19.

[10] F. Corno, M. S. Reorda, G. Squillero, A. Manzone, and A. Pincetti, âĂIJAutomatic test bench generation for validation of rt-level descriptions: an industrial experience,âĂİ in Proc. of Design Automation and Test Europe Conference, 2000, pp. 385âĂ\$̧389.

[11] J. Burnim and K. Sen, âĂIJHeuristics for Scalable Dynamic Test Generation,âĂİ in Automated Software Engineering, 2008, pp. 443âĂŞ446.

[12] J. Wegener, A. Baresel, H. Sthamer, "Evolutionary Test Environment for Automatic Structural Testing," Information and Software Technology, Special Issue devoted to the Application of Metaheuristic Algorithms to Problems in Software Engineering, 2001, vol. 43 , pp. 841-854.

[13] K. Sen, D. Marinov, and G. Agha, "CUTE: a concolic unit testing engine for C," in Proc. of the 10th European Software Engineering Conference held jointly with 13th ACM SIGSOFT international symposium on Foundations of Software Engineering, 2005, pp. 263-272.

[14] K. Gent and M. S. Hsiao, "Abstraction-based relation mining for functional test generation," IEEE VLSI Test Symposium, 2015, pp. 1-6.

[15] K. Gent and M. S. Hsiao, "Functional Test Generation at the RTL Using Swarm Intelligence and Bounded Model Checking," 22nd Asian Test Symposium, 2013, pp. 233-238. 
[16] L. Liu and S. Vasudevan, "STAR: Generating input vectors for design validation by static analysis of RTL," in Proc. High Level Design Validation Test Workshop, 2009, pp. $32-37$.

[17] L. Liu and S. Vasudevan, "Efficient validation input generation in RTL by hybridized source code analysis," Design, Automation and Test in Europe, 2011, pp. 1-6.

[18] L. Zhang, M. R. Prasad, and M. S. Hsiao, "Incremental deductive and inductive reasoning for SAT-based bounded model checking," in Proc. of the IEEE International Conference on Computer Aided Design, November 2004, pp. 502-509.

[19] P. Godefroid, N. Klarlund, and K. Sen, "DART: directed automated random testing," in Proc. of the ACM SIGPLAN conference on Programming Language Design and Implementation, 2005, pp. 213-223.

[20] M. Li, K. Gent and M. S. Hsiao, "Design validation of RTL circuits using evolutionary swarm intelligence," IEEE International Test Conference, 2012, pp. 1-8.

[21] M. S. Hsiao, E. M. Rudnick and J. H. Patel, "Sequential circuit test generation using dynamic state traversal," in Proc. of European Design and Test Conference,1997, pp. 22-28.

[22] M. S. Hsiao, E. M. Rudnick, and J. H. Patel, "Dynamic state traversal for sequential circuit test generation," ACM Trans. on Design Automation of Electronic Systems, 2000, pp. $548-565$.

[23] M. S. Hsiao, E. M. Rudnick, and Janak H. Patel, "Fast static compaction algorithms for sequential circuit test vectors," IEEE Tran. on Computers, 1999, vol. 48, no. 3, pp. 311-322. 
[24] P. McMinn and M. Holcombe, "Evolutionary testing of state-based programs," in Proc. of the 7th annual conference on Genetic and evolutionary computation (GECCO '05), Hans-Georg Beyer (Ed.), ACM, pp. 1013-1020.

[25] Q. Xiaoke and P. Mishra, "Scalable Test Generation by Interleaving Concrete and Symbolic Execution," in International Conference on VLSI Design and International Conference on Embedded Systems, 2014, pp. 104-109.

[26] R. Ferguson and B. Korel, "Software test data generation using the chaining approach," in Proc. of 1995 IEEE International Test Conference (ITC), 1995, pp. 703-709.

[27] R. Arora and M. S. Hsiao, "Enhancing SAT-based bounded model checking using sequential logic implications," in Proc.s of the IEEE VLSI Design Conference, January, 2004, pp. 784-787.

[28] S. Bagri, K. Gent and M. S. Hsiao, "Signal domain based reachability analysis in RTL circuits," Sixteenth International Symposium on Quality Electronic Design, 2015, pp. $250-256$.

[29] T. Zhang, T. Lv and X. Li, "An abstraction-guided simulation approach using Markov models for microprocessor verification," in Proc. of Design, Automation 83 Test in Europe Conference \&' Exhibition (DATE '10), 2010, pp. 484-489.

[30] T. Niermann and J. H. Patel, "HITEC: a test generation package for sequential circuits," in Proc. of the conference on European design automation (EURO-DAC '91). IEEE Computer Society Press, 1991, pp. 214-218.

[31] V. V. Acharya, S. Bagri and M. S. Hsiao, "Branch guided functional test generation at the RTL," in IEEE European Test Symposium, 2015, pp. 1-6. 
[32] Y. Zhou, T. Wang, H. Li, T. Lv and X. Li, "Functional Test Generation for Hardto-Reach States Using Path Constraint Solving," in IEEE Tran. on Computer-Aided Design of Integrated Circuits and Systems, 2016, vol. 35, no. 6, pp. 999-1011.

[33] Y. Zhou, T. Wang, T. Lv, H. Li and X. Li, "Path Constraint Solving Based Test Generation for Hard-to-Reach States," 22nd Asian Test Symposium, 2013, pp. 239-244.

[34] Microsoft Research, "Z3 theorem prover," https://z3.codeplex.com/

[35] SRI International Computer Science Laboratory, "The Yices SMT Solver," http://yices.csl.sri.com/

[36] W. Snyder, D. Galbi and P. Wasson, "Verilator," https://www.veripool.org/wiki/verilator 